\title{
Selective Applicative Functors
}

\author{
ANDREY MOKHOV, Newcastle University, United Kingdom \\ GEORGY LUKYANOV, Newcastle University, United Kingdom \\ SIMON MARLOW, Facebook, United Kingdom \\ JEREMIE DIMINO, Jane Street, United Kingdom
}

Applicative functors and monads have conquered the world of functional programming by providing general and powerful ways of describing effectful computations using pure functions. Applicative functors provide a way to compose independent effects that cannot depend on values produced by earlier computations, and all of which are declared statically. Monads extend the applicative interface by making it possible to compose dependent effects, where the value computed by one effect determines all subsequent effects, dynamically.

This paper introduces an intermediate abstraction called selective applicative functors that requires all effects to be declared statically, but provides a way to select which of the effects to execute dynamically. We demonstrate applications of the new abstraction on several examples, including two industrial case studies.

CCS Concepts: • Software and its engineering; • Mathematics of computing;

Additional Key Words and Phrases: applicative functors, selective functors, monads, effects

ACM Reference Format:

Andrey Mokhov, Georgy Lukyanov, Simon Marlow, and Jeremie Dimino. 2019. Selective Applicative Functors. Proc. ACM Program. Lang. 3, ICFP, Article 90 (August 2019), 29 pages. https://doi.org/10.1145/3341694

\section{INTRODUCTION}

Monads, introduced to functional programming by Wadler [1995], are a powerful and general approach for describing effectful (or impure) computations using pure functions. The key ingredient of the monad abstraction is the bind operator, denoted by $>>=$ in Haskell ${ }^{1}$ :

$$
(>>): \text { : Monad } f \Rightarrow f a \rightarrow(a->b) \rightarrow f b
$$

The operator takes two arguments: an effectful computation $f$ a, which yields a value of type a when executed, and a recipe, i.e. a pure function of type $a->f b$, for turning a into a subsequent computation of type $f \mathrm{~b}$. This approach to composing effectful computations is inherently sequential: until we execute the effects in $f$ a, there is no way of obtaining the computation $f b$, i.e. these computations must be performed in sequence. The ability to enforce a sequential execution order is crucial for non-commutative effects, such as printing to the terminal. Furthermore, the dependence between subsequent effects can be used for conditional effect execution, as demonstrated below.

\footnotetext{
${ }^{1}$ We use Haskell throughout this paper, but the presented ideas are not language specific. We release two libraries for selective applicative functors along with this paper, written in Haskell (https://hackage.haskell.org/package/selective) and OCaml (https://opam.ocaml.org/packages/selective). The ideas have also been translated to Coq [Lukyanov 2019], Kotlin [Gibson 2019], PureScript [Holvikari 2018], Scala [Birchall and Dickson 2019] and Swift [Ruiz-López 2019].
}

Authors' addresses: Andrey Mokhov, Newcastle University, United Kingdom, andrey.mokhov@ncl.ac.uk; Georgy Lukyanov, Newcastle University, United Kingdom, g.lukyanov2@ncl.ac.uk; Simon Marlow, Facebook, London, United Kingdom, smarlow@fb.com; Jeremie Dimino, Jane Street, London, United Kingdom, jdimino@janestreet.com.

Permission to make digital or hard copies of part or all of this work for personal or classroom use is granted without fee provided that copies are not made or distributed for profit or commercial advantage and that copies bear this notice and the full citation on the first page. Copyrights for third-party components of this work must be honored. For all other uses, contact the owner/author(s).

(C) 2019 Copyright held by the owner/author(s).

2475-1421/2019/8-ART90

https://doi.org/10.1145/3341694

Proc. ACM Program. Lang., Vol. 3, No. ICFP, Article 90. Publication date: August 2019. 
Consider a simple example, where we use the monad $f=I 0$ to describe an effectful program that prints "pong" to the terminal if the user enters "ping":

pingPongM : : IO ()

pingPongM $=$ getLine $>>=\backslash s \rightarrow$ if $s==$ "ping" then putStrLn "pong" else pure ()

The first argument of the bind operator reads a string using getLine : : I0 String, and the second argument is the function of type String -> IO (), which prints "pong" when s == "ping".

As we will see in sections $\S 3$ and $\S 4$, in some applications it is desirable to know all possible effects statically, i.e. before the execution. Alas, this is not possible with monadic effect composition. To inspect the function $\backslash \mathrm{s}->$. . , we need a string $\mathrm{s}$, which becomes available only during execution. We are therefore unable to predict the effects that pingPongM might perform: instead of conditionally executing putStrLn, as intended, it might delete a file from disk, or launch proverbial missiles.

Applicative functors, introduced by McBride and Paterson [2008], can be used for composing statically known collections of effectful computations, as long as these computations are independent from each other. The key ingredient of applicative functors is the apply operator, denoted by $\langle *\rangle$ :

$(<*>)$ : : Applicative $f \Rightarrow f(a->b) \rightarrow f a \rightarrow f b$

The operator takes two effectful computations, which - independently - compute values of types $a->b$ and $a$, and returns their composition that performs both computations, and then applies the obtained function to the obtained value producing the result of type b. Crucially, both arguments and associated effects are known statically, which, for example, allows us to pre-allocate all necessary computation resources upfront $(\S 3)$ and execute all computations in parallel (§4).

Our ping-pong example cannot be expressed using applicative functors. Since the two computations must be independent, the best we can do is to print "pong" unconditionally:

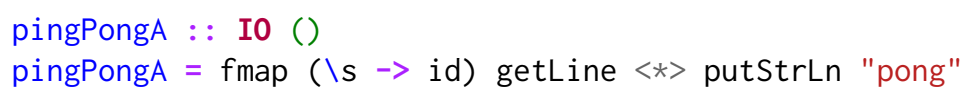

Here we use fmap ( $\backslash s->i d$ ) to replace the input string $s$, which we now have no need for, with the identity function id : : () $\rightarrow()$, thus matching the type of putStrLn "pong" : : IO (). We cannot execute the putStrLn "pong" effect conditionally but, on the positive side, the effects are no longer hidden behind opaque effect-generating functions, which makes it possible for the applicative functor $f=I 0$ to statically know the two effects embedded in pingPongA.

At this point the reader is hopefully wondering: can we combine the advantages of applicative functors and monads, i.e. allow for conditional execution of some effects while retaining the ability to statically know all effects embedded in a computation? It will hardly be a surprise that the answer is positive, but it is far from obvious what the right abstraction should be. For example, one might consider adding a new primitive called whenS to I0:

whenS : : I0 Bool $\rightarrow$ IO () $\rightarrow$ IO ()

This primitive executes the first computation, and then uses the obtained Bool to decide whether to execute the second computation or skip it. Let us rewrite the ping-pong example using whens:

pingPongS : : IO ()

pingPongS $=$ whenS (fmap (=="ping") getLine) (putStrLn "pong")

We replace the input string $s$ with True if it is equal to "ping", and False otherwise, thereby appropriately selecting the subsequent effectful computation. This approach gives us both conditional execution of putStrLn "pong", and static visibility of both effects (see §5.2). Crucially, whenS must be an I0 primitive instead of being implemented in terms of the monadic bind (>>=), because the latter would result in wrapping putStrLn "pong" into an opaque function, as in pingPongM. 
The main idea of this paper is that whenS, as well as many other similar combinators, can be seen as special cases of a new intermediate abstraction, called selective applicative functors, whose main operator for composing effectful computations is select:

select : : Selective $f \Rightarrow f($ Either $a b) \rightarrow f(a->b) \rightarrow f b$

Intuitively, the first effectful computation is used to select what happens next: if it yields a Left a you must execute the second computation in order to produce $a b$ in the end; otherwise, if it yields a Right b, you may skip the subsequent effect, because you have no use for the resulting function. Note the possibility of speculative execution: in some contexts, we can execute both computations in parallel, cancelling the second computation if/when the first one evaluates to a Right $b$.

The contributions of this paper are as follows:

- We introduce selective applicative functors as a general abstraction situated between applicative functors and monads, characterising the relationships between all three abstractions with a set of laws, and defining a few important instances (\$2).

- We discuss applications of the abstraction on two industrial case studies: the OCaml build system Dune [Jane Street 2018] (§3) and Facebook’s Haxu library [Marlow et al. 2014] (§4).

- We present free selective applicative functors and show how to use them to implement embedded domain-specific languages with both conditional effects and static analysis (§5).

We discuss alternatives to selective applicative functors and related work in sections $\S 6$ and $\S 7$.

\section{SELECTIVE FUNCTORS}

In this section we introduce selective applicative functors, which we will subsequently refer to as simply selective functors, for brevity. We start by defining the new abstraction, and then use it in $\S 2.1$ to implement several derived combinators, such as the aforementioned whenS. In $\S 2.2$ we provide several examples of selective functors, and further discuss the relationships between applicative functors, selective functors, and monads. In §2.3, these relationships are further elaborated and expressed as a set of laws that all selective functors are required to satisfy.

Like applicative functors [McBride and Paterson 2008], selective functors provide a way to embed pure values into an effectful context $f$ using the function pure, and give meaning to composition of two independent effectful computations using the operator $\langle *\rangle$. See Fig. 1 for the standard definition of the corresponding type class Applicative. Selective functors enrich the applicative interface with the select method, which gives meaning to the composition of two effectful computations, where, in contrast to $\langle *\rangle$, the second computation depends on the first one:

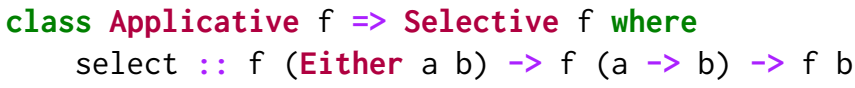

One can think of select as a selective function application: parametricity [Wadler 1989] dictates that, when given a Left $a$, we must execute the effects in $f(a->b)$, apply the obtained function to $a$, and return the resulting $b$; on the other hand, when given a Right b, we may skip the effects associated with the function, and return the given $b^{2}$.

Following the notational convention for applicative operators, we also define the left-associative infix operator alias $<*$ ? for select: the angle bracket pointing to the left means we always use the corresponding value; the value on the right, however, may be skipped, hence the question mark.

One can implement select using monads in a straightforward manner: examine the value produced by $f$ (Either $a b$ ) with the bind operator, and then, in the Left a case, execute the

\footnotetext{
${ }^{2}$ Note, however, that if $\mathrm{f}$ a holds no values of type a, i.e. a is a phantom type variable [Leijen and Meijer 2000], then the effects in $f(a->b)$ can be skipped unconditionally. The selective functor Under is a good example (see §2.2).
} 
Table 1. Comparison of apply, select and bind operators in terms of their expressive power. We discuss static analysis in $\$ 2.2$ and §3; parallelism and speculative execution in §4; conditional and arbitrary dynamic effects in $\S 2.1$ (whenS) and $\S 5.2$ (greeting). Note that each operator has one unique ability that the two others lack.

\begin{tabular}{l||c|c|c} 
Notions that can be expressed using an operator & apply $(\langle *>)$ & select $(<*$ ?) & bind (>>=) \\
\hline Arbitrary dynamic effects & & & $\checkmark$ \\
Conditional execution of effects & & $\checkmark$ & $\checkmark$ \\
Speculative execution of effects & $\checkmark$ & $\checkmark$ & \\
Static visibility and analysis of effects & $\checkmark$ & &
\end{tabular}

Any Applicative instance can thus be given a Selective instance. The opposite is also true in the sense that one can recover the operator $\langle *\rangle$ from select as follows:

apS : : Selective $f \Rightarrow f(a \rightarrow b) \rightarrow f a \rightarrow f b$

apS $f x=\operatorname{select}($ Left $\langle \$\rangle f)((\&)\langle \$>x)$

Here we tag the function $a->b$ with Left and apply the reverse function application (see Fig. 2) to the value a, thus matching the type signature of select. Since the Right case is impossible, the effect $x:: f a$ is executed unconditionally. Note, however, that in general $\langle *\rangle$ and apS are not equivalent. Selective functors that satisfy the property $(\langle *\rangle)=$ apS will be called rigid; they will turn out to have a simple normal form, which we will exploit in the free construction in $\S 5$.

It is worth emphasising that the subclass relationships Applicative $f=>$ Selective $f$ and Applicative $f \Rightarrow$ Monad $f$ are different. Some applicative functors are not monads, e.g. the Const functor (\$2.2), but every applicative functor is also a selective functor, as witnessed by the function selectA. The subclass relationship Applicative $f \Rightarrow$ Selective $f$ is justified only by the extra method select in Selective. While select = selectA is a valid implementation of select, it is not the only useful implementation, as will be demonstrated in §2.2. The applicative-selective-monad hierarchy therefore reflects method set inclusion: $\{\langle *\rangle\} \subset\{\langle *\rangle$, select $\} \subset\{\langle *\rangle$, select, $\rangle\rangle=\}$. Table 1 compares the three methods in terms their expressive power. Different applications require different sets of methods; for example, as we will see in $\S 4$, HAxL requires all three: $\langle *\rangle$ for parallelism, select for speculative execution, and $\gg>=$ for arbitrary dynamic effects.

We will come back to the relationship between applicative functors, selective functors and monads in $\S 2.3$, after first exploring selective combinators that can be written using the selective interface (§2.1), and then looking at some concrete examples of selective functors (§2.2).

(\&)

(.)

id

const

flip

uncurry : : $(a \rightarrow b \rightarrow c) \rightarrow(a, b) \rightarrow c$

foldr :: (a $->b \rightarrow b) \rightarrow b->[a]->b$

bool $\quad:$ : a $\rightarrow$ a $\rightarrow$ Bool $\rightarrow$ a

maybe $\quad:: \mathrm{b} \rightarrow(\mathrm{a} \rightarrow \mathrm{b}) \rightarrow$ Maybe $\mathrm{a} \rightarrow \mathrm{b}$

either $::(a->c) \rightarrow(b \rightarrow c) \rightarrow$ Either $a b \rightarrow c$

first $\quad:(a->c) \rightarrow$ Either $a b \rightarrow$ Either $c b$

bimap $\quad::(a->c) \rightarrow(b->d) \rightarrow$ Either $a b \rightarrow$ Either $c d$

void : : Functor $f \Rightarrow f$ a $\rightarrow f($ )
-- Function application

-- Reverse function application

-- Function composition

-- Identity function

-- Constant function

-- Flip function arguments

-- Uncurry a function

-- Reduce a list to a value

-- Deconstruct a Bool

-- Deconstruct a Maybe

-- Deconstruct an Either

-- Map over Left

-- Map over Left and Right

-- Discard an effect's value

Fig. 2. Type signatures and descriptions of standard operators and functions used throughout the paper. 


\subsection{Selective Combinators}

As a first use-case of the interface provided by selective functors, let us revisit our ping-pong example from $\S 1$ and implement the combinator whenS:

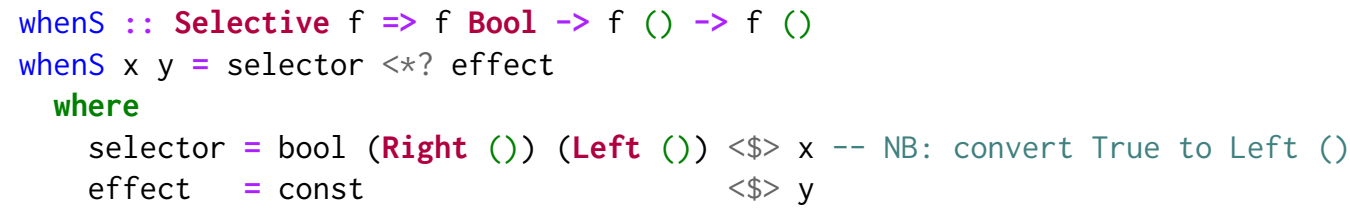

We first bring the given effectful computations into the right shape by using the Functor's map operator. Specifically, $x:: f$ Bool is converted into the selector : : $f$ (Either () ()), and $y:: f()$ is converted into the effect : : $f(() \rightarrow())$. The results are composed using the select operator $<*$ ?, and the meaning of this composition is determined by the supplied Selective $f$ instance. For example, an instance like $f=I 0$ would skip y if $x$ yields False, as exploited by our implementation of pingPongS. On the other hand, instances used for static analysis would record both $\mathrm{x}$ and $\mathrm{y}$ as possible effects. See more examples in $§ 2.2$.

It is worth noting that unlike the select operator, whose implementation is almost completely determined by parametricity (i.e., the only real question is: “To skip, or not to skip?"), whenS admits a variety of (incorrect) implementations. In particular, due to Boolean blindness ${ }^{3}$, it is easy to inadvertently implement unlesss, which has the same type but flips the meaning of the Boolean value. The ability to reason parametrically was one of the guiding principles we used when looking for a good abstraction for selective functors: select provides this ability, whereas whens does not.

A strong contender for playing the leading role in selective functors is the function branch that, given an effectful computation $x:: f$ (Either $a b)$, selects which of the two subsequent computations, namely $1:: f(a->c)$ or $r:: f(b->c)$, to execute:

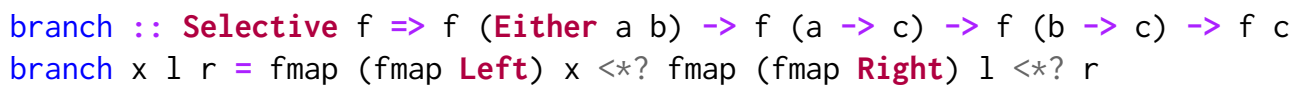

While we encourage the reader to derive an implementation of branch as an exercise, we would like to share our intuition behind it, as it will be useful for free selective functors in $§ 5$. The select operator allows us to eliminate one of the cases in a sum type, namely the Left a case in Either a b, leaving the other case intact. To implement branch, we will need to apply $<*$ ? twice, eliminating $a$ and $b$ one after another. The first application is tricky because $f($ Either $a b)$ and $f(a->c)$ do not match the type signature of $<*$ ? . To fix the mismatch, we convert them to $f$ (Either a (Either $b c$ )) and $f(a->$ Either $b c)$, respectively. The second application of $<*$ ? is then straightforward.

As will be discussed in $§ 6.1$, we could have chosen to use branch instead of select as the method of the Selective type class. Our choice of select follows the Occam's razor principle: select is simpler than branch, which, in particular, leads to a simpler free construction (§5.1).

By instantiating select with $a=b=()$ we have earlier obtained whenS. Below we repeat the exercise with branch, obtaining another familiar conditional combinator ifs:

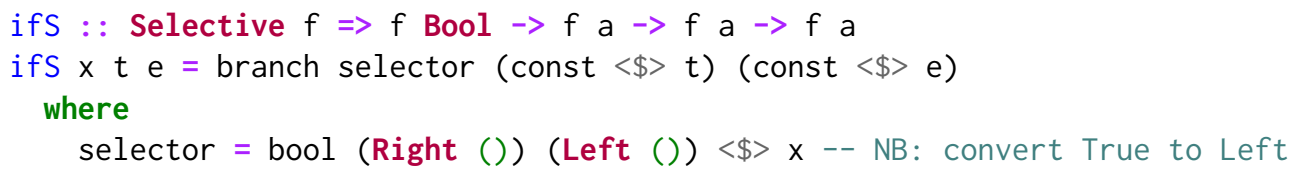

Many conditional combinators, which are typically associated with the Monad type class, can be expressed using selective functors, as shown in Fig. 3, making them reusable in new contexts. In

\footnotetext{
${ }^{3}$ The term refers to the fact that the True and False values are not distinguished at the type level, see Harper [2011].
} 


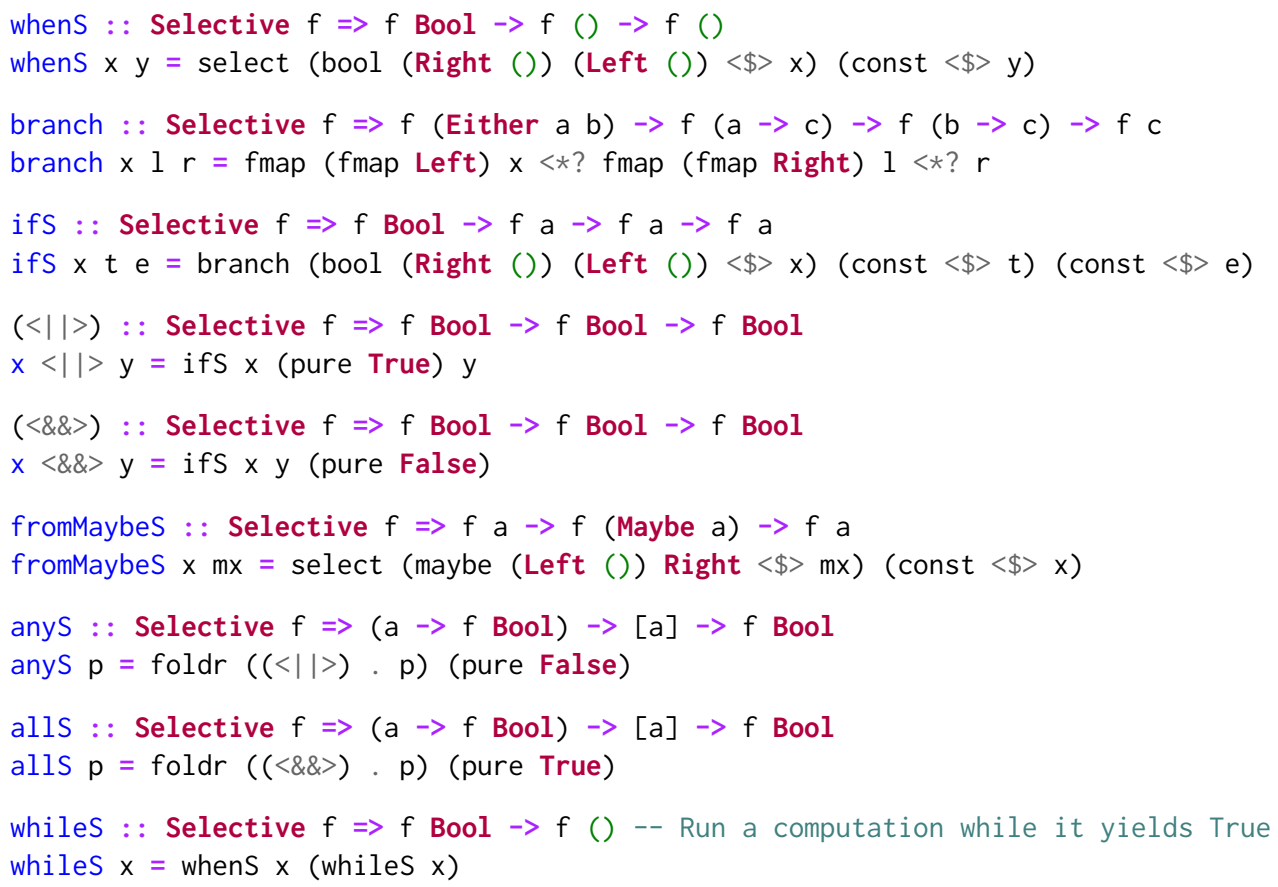

Fig. 3. A library of selective combinators. The names and order of parameters are inherited from the standard Haskell library. For example, fromMaybeS corresponds to the standard fromMaybe : : a $->$ Maybe a $->$ a and retains the short-circuiting behaviour, i.e. if the second argument yields a Just, the first argument is skipped.

particular, the logical combinators $\langle||\rangle$ and $\langle \& \&\rangle$ will play an important role in improving the efficiency of the HAXL framework in $§ 4$. To emphasise the monadic flavour of selective functors, we can use ifS to implement the bind operator specialised to Bool:

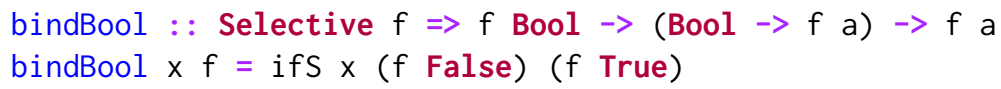

This can be achieved not only for Bool, but for any enumerable type, as we will discuss in $§ 6.1$.

\subsection{Examples of Selective Functors}

Having explored various useful combinators that can be implemented on top of the minimalistic selective interface, in this section we look at several examples of selective functors.

As we have observed at the beginning of this section, any monad can be given a Selective instance simply by using select $=$ selectM as the definition. As an example, below we define a selective instance for I0, and test the function pingPongS from $\S 1$ in an interactive GHC session:

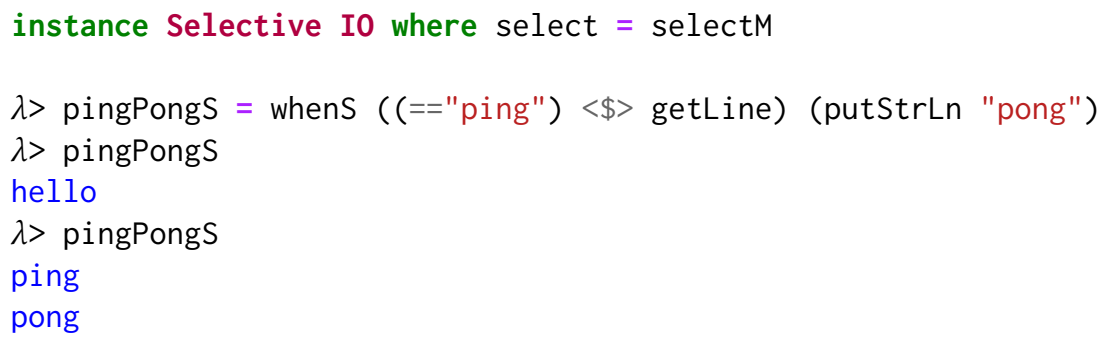


As desired, the effect putStrLn "pong" occurs conditionally. In $§ 5.2$ we will see how to statically analyse pingPongS, i.e. obtain a list of all possible effects before the execution, which can be done by interpreting pingPongS in the selective functor Over, introduced below.

We first recall the functor Const $\mathrm{m}$ a, which is an interesting and surprisingly useful instance of the Applicative type class. It stores no values of type a, but keeps track of the applicative computation structure in the monoid value of type $\mathrm{m}$ :

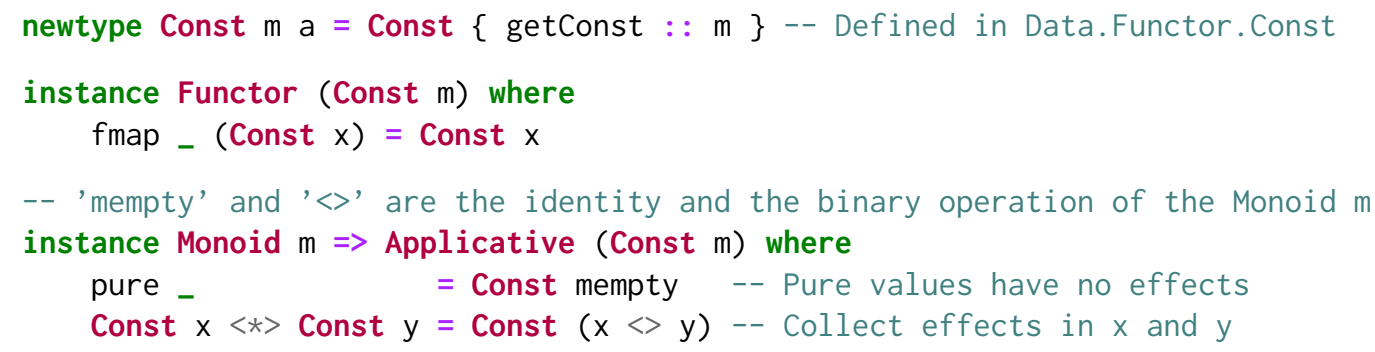

It turns out there are two useful selective instances for Const. To disambiguate between them, we will call them Over and Under, reusing ${ }^{4}$ the above Functor and Applicative instances:

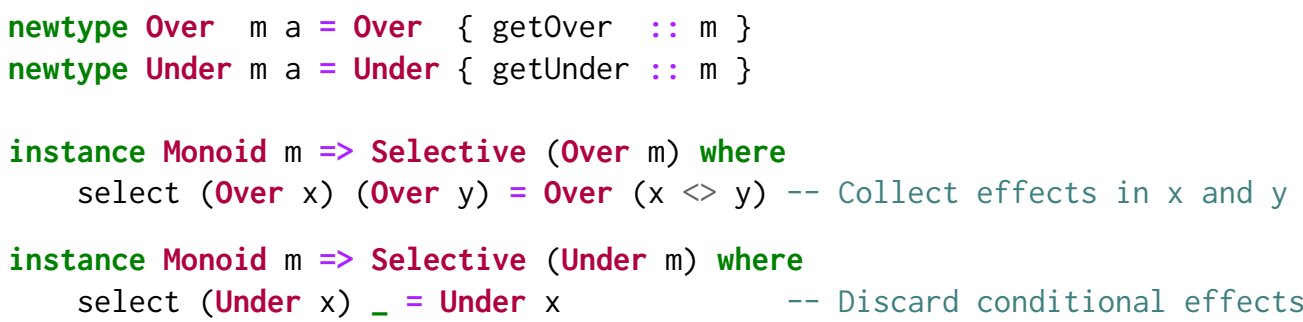

The selective functor Over can be used for computing a list of all effects embedded in a computation, i.e. an over-approximation of the effects that will actually occur. This is achieved by keeping track of effects in both arguments of the select operator. The selective functor Under, on the other hand discards the second argument of select, and therefore computes an under-approximation, i.e. a list of effects that are guaranteed to occur. Let us give these two instances a try:

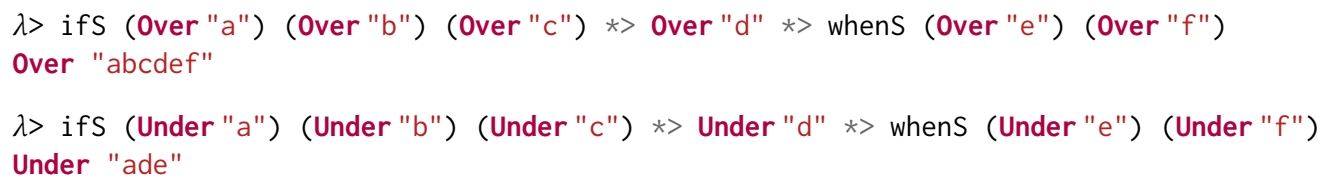

As expected, Over collects all effects, whereas Under does not look beyond "opaque" conditions. A deeper difference between them is that Over is a rigid selective functor, i.e. $(\langle *\rangle)=a p S$, but Under is not. Indeed, Under "a" <*> Under "b" records both "a" and "b", but apS (Under "a") (Under "b") records just "a" because apS is implemented via select and therefore lacks information about the independence of the two effects. Intuitively, non-rigid selective functors have a richer structure due to the fact that $\langle *\rangle$ is not expressible via select.

Our last example in this section is the selective functor Validation ${ }^{5}$, which is useful for validating complex data: if reading one or more data fields has failed, all errors are accumulated (using the operator $<>$ from the semigroup e) to be reported together.

\footnotetext{
${ }^{4}$ Fortunately, thanks to the new GHC extension DerivingVia [Blöndal et al. 2018], we can reuse Const instances without duplicating any code, simply by adding deriving (Functor, Applicative) via (Const $\mathrm{m}$ ) to the newtype definitions. ${ }^{5}$ Applicative functors Const and Validation appeared under the names Accy and Except in [McBride and Paterson 2008].
} 


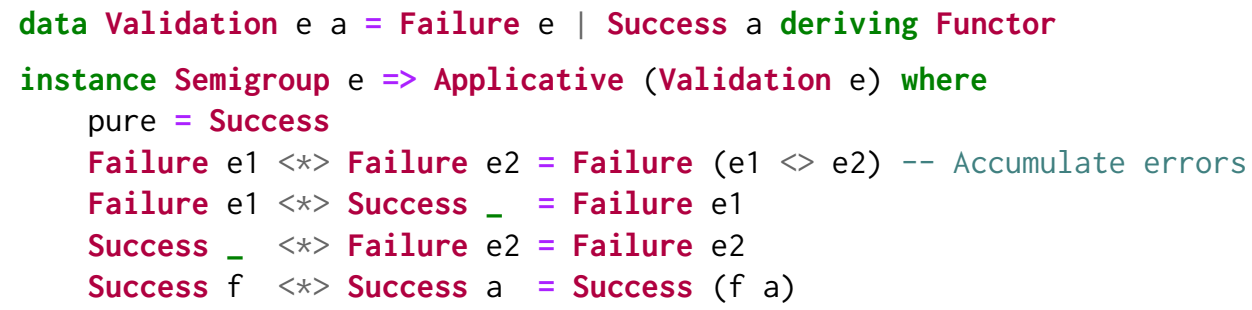

The error-accumulating behaviour cannot be extended to a Monad instance: after the first Failure we have no value to pass to the second argument of the bind operator. We can, however, define the following Selective instance, which allows us to validate data in the presence of conditions.

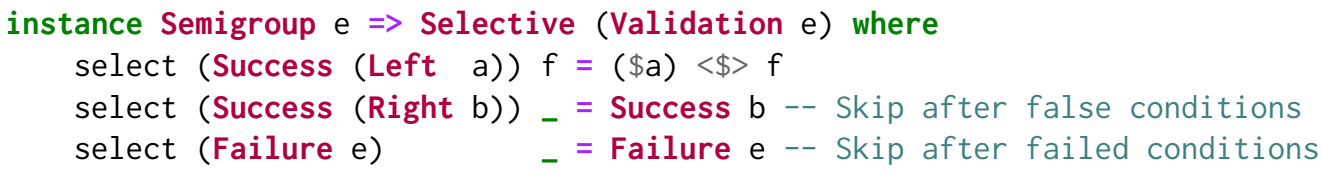

Similarly to Under, we discard the second argument but only if the first has resulted in a Right value or failed. This allows us not to report validation errors in inactive branches or in branches hidden behind failed conditionals (the corresponding fields are not necessarily needed).

Below we define a function that constructs a shape (a circle or a rectangle) given a choice of the shape $x$, and the shape's parameters $(r, w$, and $h$ ) in an arbitrary selective functor $f$. You can think of the inputs as results of reading the corresponding fields from a web form, where $\mathrm{x}$ is a checkbox, and all other fields are numeric textboxes, some of which may be empty.

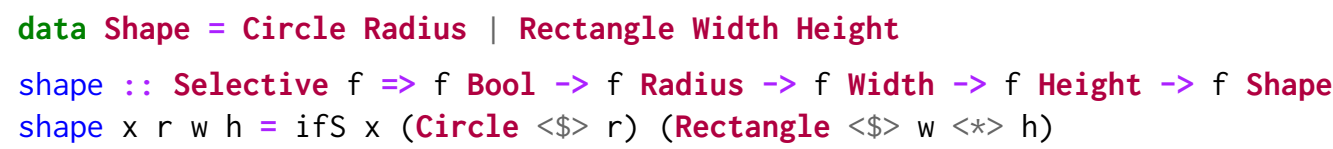

We choose $f=$ Validation [String] to report the errors that occurred when reading values from the form. Let us see how this works.

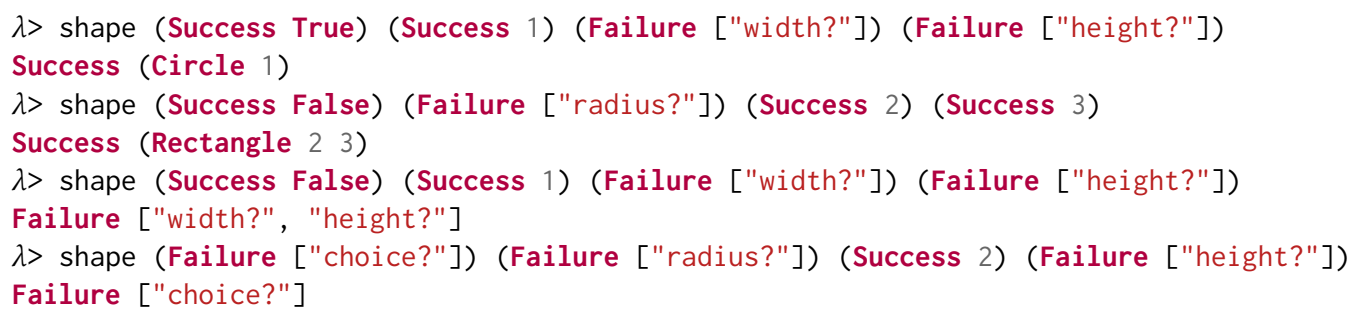

In the last example, since the shape's choice could not be read, we do not report any subsequent errors. But it does not mean we are short-circuiting the validation: we will continue accumulating errors as soon as we get out of the failed conditional, as demonstrated below.

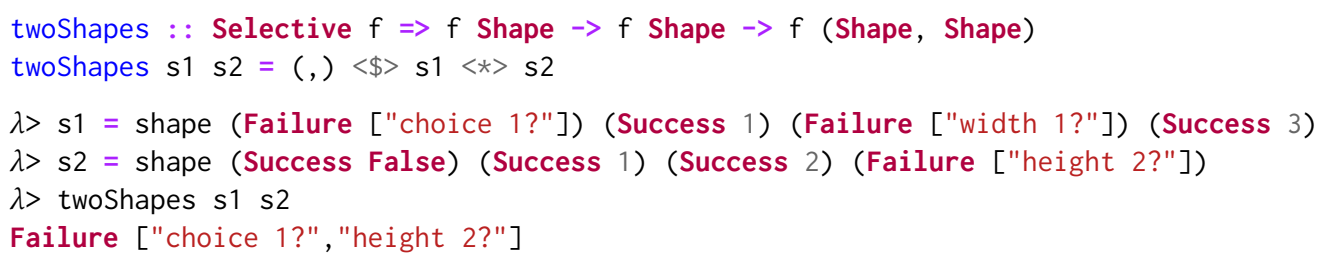

Like Under, the Validation instance is not a rigid selective functor because select occasionally discards effects in the second argument. 


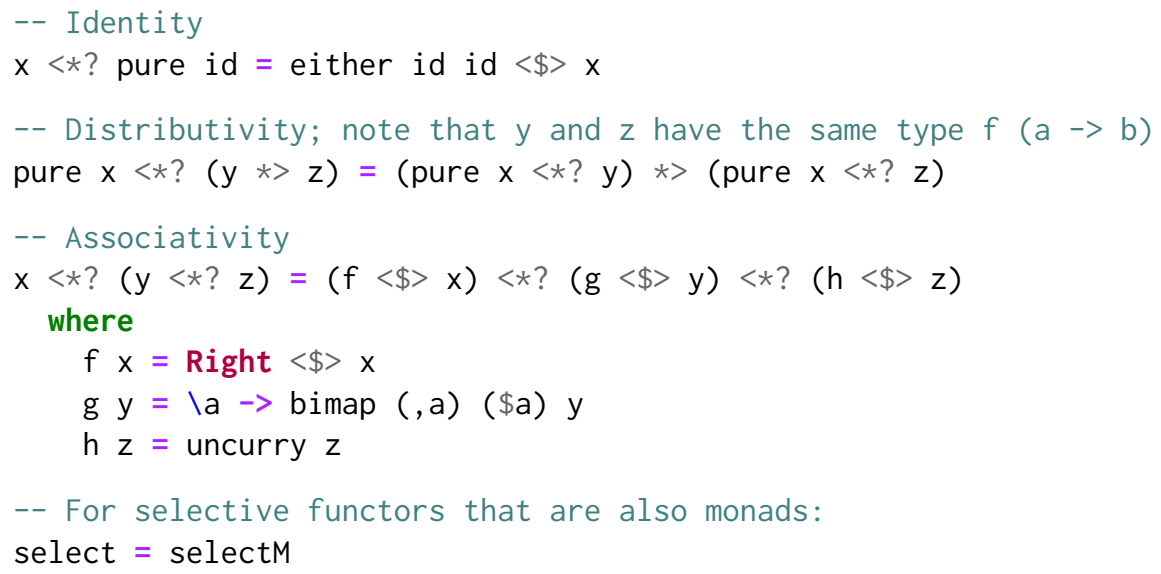

Fig. 4. Laws (top) and theorems (bottom) of selective functors. Coq proofs that the selective instances from $\$ 2.2$ are lawful are available in the supplementary material.

\subsection{Laws}

Now that we have seen several instances of selective functors, it is time to discuss the laws that we expect these instances to satisfy. In particular, it is very useful to know how the selective interface interacts with existing applicative and monadic interfaces. Real code might mix all of these abstractions, because each of them is useful in its own right, and the laws presented in this section allow us to safely refactor such code while keeping its original meaning.

Fig. 4 lists all laws for selective functors, as well as some useful theorems that can be derived from these laws and parametricity [Wadler 1989]. Below we discuss them in order.

The identity and distributivity laws determine the interaction of the select operator with pure computations. It should be impossible to distinguish $x<*$ ? pure id from a direct execution of $x$ followed by the extraction of a value from the obtained Either a a. In particular, the select operator is not allowed to duplicate effects associated with $x$. Similarly, the select operator is not allowed to sneak in any effects if the first computation is pure, which allows pure $x<*$ ? to be distributed through the applicative sequencing operator $*>$. When applied in reverse, the distributivity law allows us to simplify sequences of conditional operations as long as the conditions are pure. The generalised identity theorem follows from the identity law by parametricity. 
Note that it is not a requirement for selective functors to skip unnecessary effects. In particular, we do not require that pure (Right $x$ ) $<*$ ? $y=$ pure $x$. It may be counterintuitive, but omitting this law makes selective functors more useful. Typically, when executing a selective computation, you would want to skip the unnecessary effects (saving work); but on the other hand, if your goal is to statically analyse a given selective computation and extract the set of all possible effects (without actually executing them), then you do not want to skip any effects, because this would defeat the purpose of static analysis. Over is an example of a selective functor that would violate the requirement to skip unnecessary effects. Similarly, we do not require that pure (Left $x)<*$ ? $y=(\$ x)<\$>$ y thus legalising under-approximating instances like Under. It is worth noting, however, that the monadic select operator selectM does satisfy the pure Left and pure Right properties.

Such loose requirements on select with respect to unnecessary effects might seem troublesome. Below we list three reasons why we chose to keep the requirements loose.

- Requiring unnecessary effects to be skipped rules out any instances that could be used for static analysis. Indeed, the only way to obey this law would be to look at actual values at runtime, since it is impossible to statically know if $f$ (Either $a b$ ) contains a Right value.

- One might suggest having two classes Selective and StrictSelective, the latter with stricter requirements. But this second class would be useless: to statically analyse a computation you would have to express it via Selective, since it is inhabited by instances like Over. As for the execution, a Monad is perfectly suitable, as we will see in §5.2.

- Finally, there is a good precedent: the Applicative type class has no requirements on the order in which effects are executed: left-to-right, right-to-left, or in parallel. This loose specification allows some instances to execute effects sequentially (typically from left to right), and other instances to execute effects in parallel. Note that as soon as we also have a Monad, we gain an additional requirement $(\langle *\rangle)=$ ap, which tells us that, at the semantic level, the result should be as if the effects were executed in sequence from left to right. We follow the same approach by requiring select = selectM if $\mathrm{f}$ is also a Monad (see below).

The associativity law states that it should always be possible to re-associate a sequence of select operators to the left, by doing the necessary adjustments to the shapes of the inner values. These adjustments, called $\mathrm{f}, \mathrm{g}$, and $\mathrm{h}$ in Fig. 4, are admittedly obscure and require an explanation. For the expression $x<*$ ? $(y<*$ ? $z)$ to typecheck, the arguments should have the following types:

$$
x:: f \text { (Either } a b) \quad y:: f(\text { Either } c(a-b)) \quad z:: f(c \rightarrow a-b)
$$

On the other hand, the resulting expression $p<*$ ? $q<*$ ? $r$ has arguments of these types:

$$
p:: f \text { (Either a (Either (c,a) b)) } q:: f(a->\text { Either }(c, a) b) \quad r:: f((c, a) \rightarrow b)
$$

To adjust $x$, we inject it in a larger sum type; $y$ is turned into a function accepting a value of type a from $p$; and $z$ is simply uncurryed. As we will see in $\S 6.2$, the associativity law can be expressed much more naturally if we switch to a more symmetric select operator. A similar phenomenon occurs with the composition law of the Applicative type class when the latter is expressed using an equivalent but more symmetric Monoidal interface [McBride and Paterson 2008].

The final law links selective functors to monads: in the spirit of the conventional applicativemonad law $(\langle *\rangle)=$ ap, we require that monadic instances implement select so that it is extensionally equivalent to selectM, which in particular means that unnecessary effects are skipped. A consequence of this law is that monadic selective functors are also rigid, i.e. $(\langle *\rangle)=\mathrm{apS}$, which makes it practically feasible to reason about code written using all three abstractions.

Fig. 4 also lists a few theorems that are useful when working with selective functors. The first three come for free from parametricity: they tell how one can reshape pure contents of selective functors. Last but not least, the interchange property is a consequence of associativity and $(\langle *\rangle)=\operatorname{apS}$, which allows us to move computations inside the condition argument of the select operator. This 
property does not always hold for non-rigid selective functors: while Under respects it, Validation does not, as demonstrated by the following example:

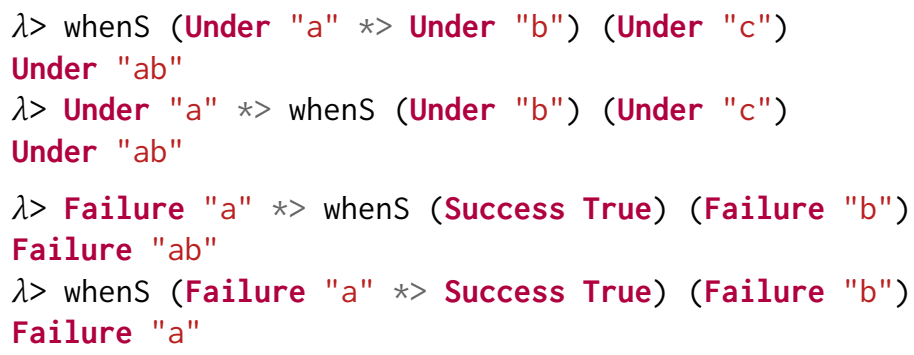

One example where the interchange law appears naturally is parser combinators, where it allows us to refactor parsers with choice, see $§ 7.2$.

\section{STATIC ANALYSIS}

In this section we discuss a real-life application that benefits from static analysis of effectful computations - the Dune build system [Jane Street 2018]. We start by introducing Dune and motivating the need for static analysis with over-approximation (§3.1), and then show how one can implement static analysis of build system dependencies using selective functors (§3.2).

\subsection{Dune Build System}

DUNE was originally developed at Jane Street and has by now become a standard build system for OCaml packages [Jane Street 2018]. At the time of writing, more than 1000 OCaml packages are using DunE as the build system. The original motivation for developing DunE (earlier known as jbuilder) was to make it easier to open source code developed in an industrial environment, and so DUNE was not meant to be used for everyday software development. However, DunE's ability to extract maximum parallelism from build scripts meant it was faster than existing build systems, such as OCAMLBUILD, and it quickly became popular, with major projects switching to Dune, for example, the CoQ proof assistant [Bertot and Castéran 2013].

One unusual feature of DUNE is the ability to statically over-approximate all build dependencies of a package. This is used at Jane Street to automatically produce package manifest files for more than 100 packages instead of maintaining them by hand. Package manifest files are consumed by package managers, such as OPAM [The OPAM team 2018], which download and install all required dependencies before the build starts.

To generate a manifest file automatically Dune needs to analyse the build graph statically, i.e. without actually running any build commands, because at this point the project cannot yet be built (due to missing dependencies). Package dependencies can be conditional and depend on values that can only be computed during the build, therefore in many situations it is impossible to statically compute an exact set of dependencies, and hence an over-approximation is used instead.

In general, one can view such static dependency analysis as a function from a build script to a set of package dependencies, and implement it directly by parsing the script and extracting all possible dependencies from it. DunE adopts a different approach: it reuses the existing script execution engine that executes build commands, but in a mock environment where commands are skipped, but their dependencies are recorded in all branches of conditional statements. By doing static analysis at this level, one can reuse a lot of code, e.g. for parsing and interpreting build scripts.

In this mock environment, some parts of the code cannot be fully evaluated as they need the output produced by external commands. However, these parts still need to be analysed. To achieve this, the original implementation of DuNE uses the arrow abstraction discussed in §7.1. To evaluate 
suitability of selective functors for this task, we have successfully prototyped an alternative core for DunE, which uses applicative and selective functors instead of arrows.

\subsection{Static Analysis of Build Dependencies}

DUNE is written in OCaml, and we therefore developed an OCaml library for selective functors. In this section, however, we choose to continue using Haskell to avoid confusion.

We follow the approach by Mokhov et al. [2018] for modelling build tasks, where a single task is represented as a higher-order function parameterised by the type of keys $\mathrm{k}$, e.g. file names, and the type of values $v$, e.g. file contents. A task takes a callback of type $k \rightarrow f v$, that the task can use to find values of its dependencies, and returns the result embedded in a selective context $f$ :

newtype Task $k v=\operatorname{Task}\{$ run : : forall $f$. Selective $f \Rightarrow(k \rightarrow f v) \rightarrow f v$ \}

The task needs to be polymorphic over $f$ so that it can be run both in build mode, by actually executing build commands, and in the mock mode, where build commands are skipped but dependencies are recorded, as explained in §3.1. For example, to compute over- and under-approximation of build dependencies we run the task in selective functors $f=$ over $[k]$ and $f=$ Under $[k]$, respectively:

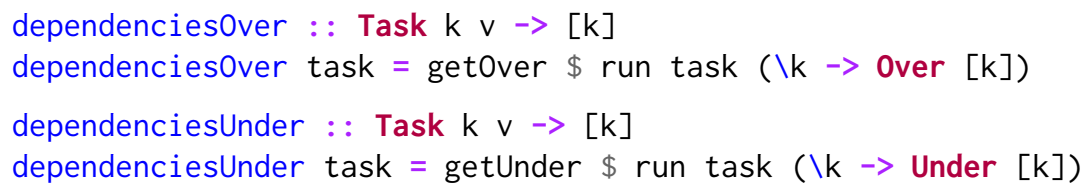

Thanks to the polymorphism of Task over $f$, we can "execute" a given task with a mock callback like $(\backslash k->0$ ver $[k]):: k->$ Over $[k] \vee$, whose only effect is recording the given key.

To demonstrate this on an example, we need a way to model a build script, i.e. a collection of build tasks. One simple approach [Mokhov et al. 2018] is to use a function that, given a key k returns either the corresponding build Task or Nothing to indicate that this key is an input (external) dependency that cannot be built and should therefore be available before the build starts:

\section{type Script k v = k $\rightarrow$ Maybe (Task k v)}

Now we have all the ingredients for creating a simple build script comprising two tasks: (i) the top-level task for building release.tar by archiving the file LICENSE and the executable exe; and (ii) the task for compiling the executable from the source src.ml and one of the two libraries: lib.c or lib.ml, depending on the configuration option stored in the config file (it is common to use an optimised low-level C implementation of a performance-critical function, falling back to high-level OCaml implementation if the former is unavailable on the system):

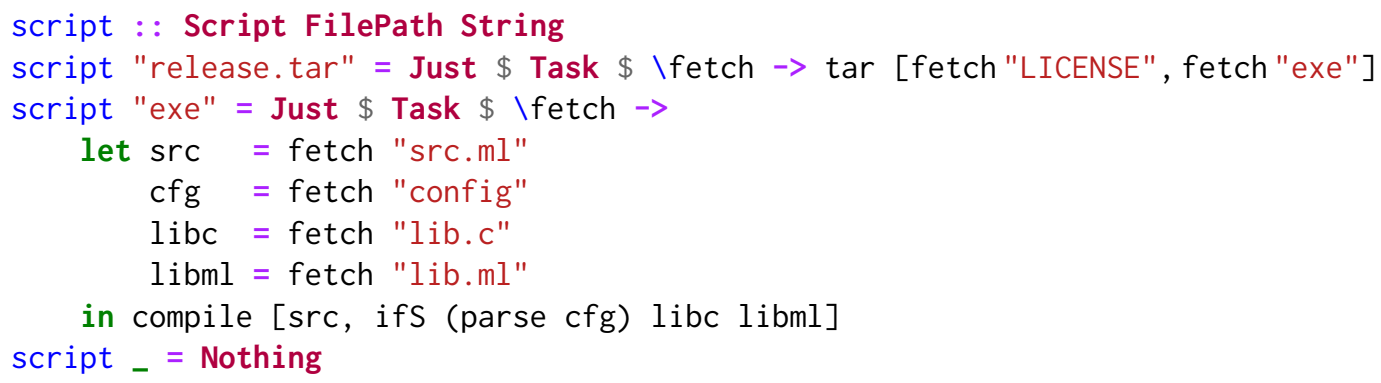

Functions tar, compile : : Selective $f \Rightarrow$ [f String] $->f$ String create an archive and compile an OCaml executable from sources/libraries, while parse : : Selective $f=>f$ String $\rightarrow f$ Bool parses a configuration file; their implementation is irrelevant for our purposes. 


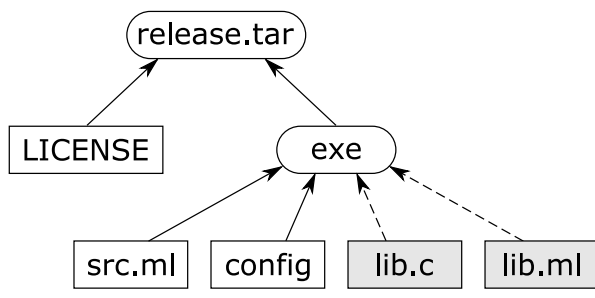

Fig. 5. An example build dependency graph. Input files are shown in rectangles, intermediate and output files are shown in rounded rectangles. Conditional dependencies are highlighted with dashed lines.

By analysing individual build tasks using dependenciesOver and dependenciesUnder, we can construct a dependency graph, where some of the dependencies are conditional, see Fig. 5:

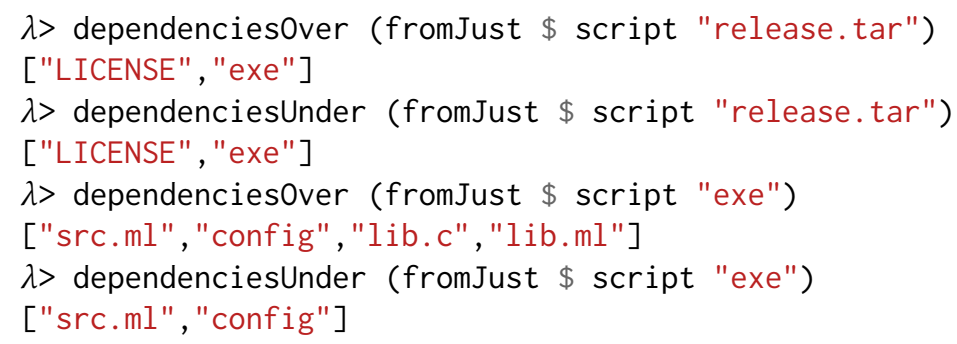

Note that while over-approximation is useful for installing all possible dependencies before the build, under-approximation is useful for maximising parallelism during the build: for example, if all input files are actually generated by running a text preprocessor, then we can start the three preprocessing tasks that are definitely needed (LICENSE, src.ml, config) in parallel, i.e. without waiting for the outcome of parsing the config file.

Applicative and monadic build systems studied in [Mokhov et al. 2018] cannot support such overand under-approximating static analysis, and the associated abstractions are therefore unsuitable for DunE. This explains why Dune developers have chosen to use the arrow abstraction ( $\$ 7.1)$. As our case study and the developed prototype demonstrate, selective functors provide a viable alternative to arrows in the context of build systems.

\section{SPECULATIVE EXECUTION}

HAXL [Marlow et al. 2014] is a framework for efficiently executing code that fetches data from external sources, typically databases or remote services. The HAxL framework allows code written in a natural style using Applicative and Monad combinators to run efficiently, by automatically parallelising the data fetch operations and batching together multiple fetches from the same data source. HAXu has been in use at Facebook, at scale, for several years now in a system that proactively detects and remediates various forms of abuse. HAXL allows the engineers working on the anti-abuse code to write clear and concise application logic, because the framework abstracts away from the details of concurrency and efficient data fetching.

To illustrate the idea using a fragment of the example code by Marlow et al. [2014], suppose we are writing the code to render a blog into HTML. The blog consists of a set of posts, where each post is identified by a PostId. The data for the blog is stored in a remote database, and the API for fetching the data from the database is as follows:

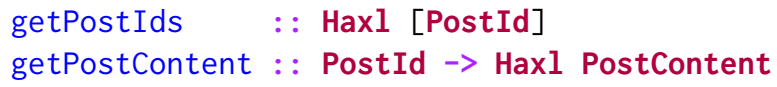




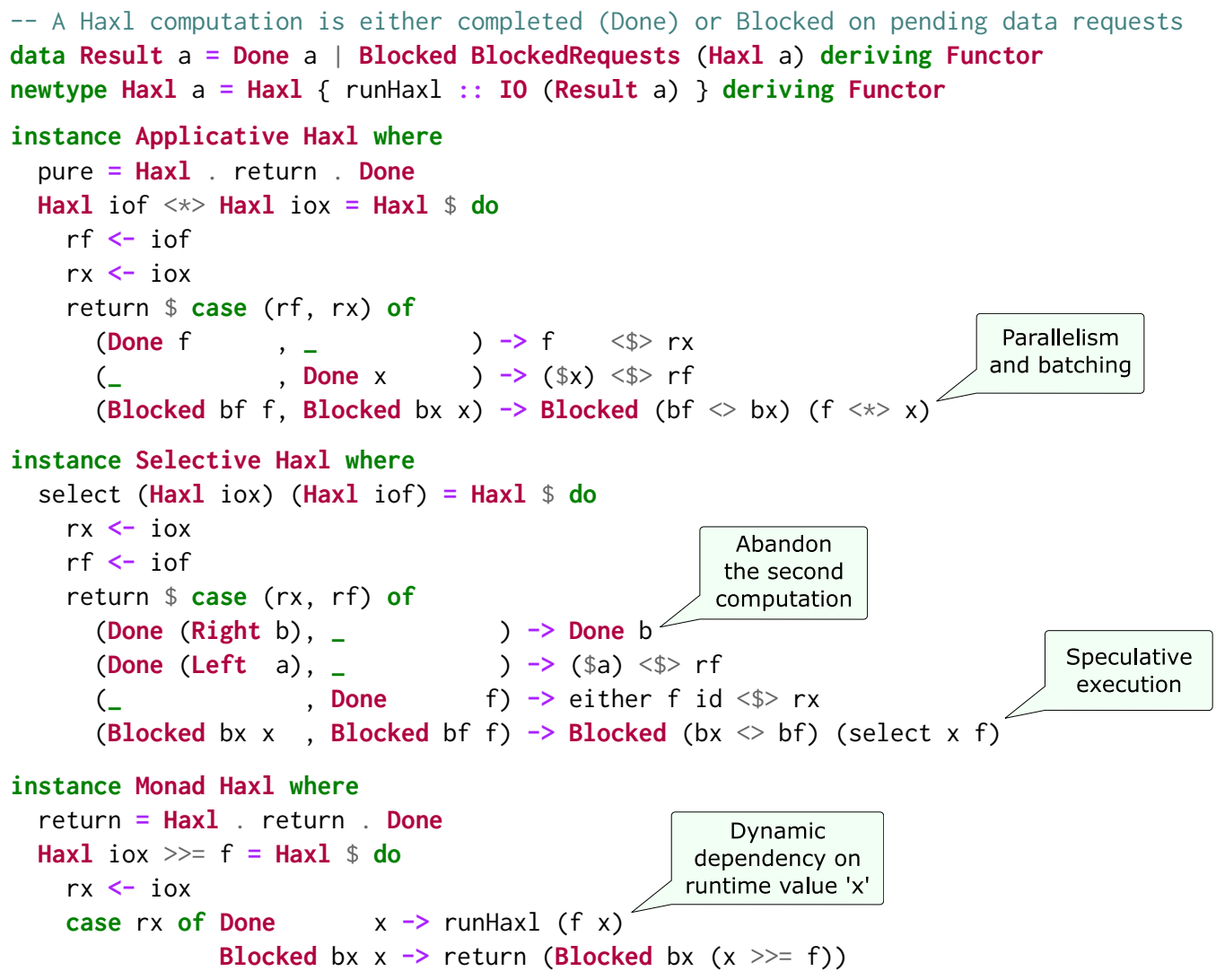

Fig. 6. An implementation of Applicative, Selective and Monad instances for the HAXL monad.

We can fetch the set of all PostIds using getPostIds, and we can fetch the content of one post using getPostContent. To get the content of all posts we could write:

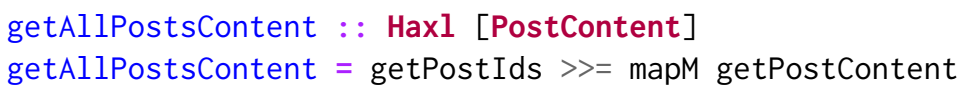

Now, when we mapM getPostContent we would really like the database queries to happen in parallel, because there are no dependencies between them. Furthermore, we might even be able to batch up the queries into a single request to the remote database.

These optimisations are performed automatically by HAXL, using a special Applicative instance that exploits the lack of dependency between the two computations to explore the computations and collect the data fetch requests that can be performed in parallel or batched together. Fig. 6 shows an implementation adapted from the code by Marlow et al. [2014]. For the purposes of the presentation here we have renamed Fetch to Haxl and omitted the exception-handling code. The key piece of HAXL's design is the Blocked/Blocked case, where two independent sets of BlockedRequests are combined together (the semigroup operator $\langle>$ is just a customised set union). HAXL also has a Monad instance, also shown in Fig. 6, which provides support for dynamic data fetches that are based on results obtained earlier. Such dynamic data fetches are sequentialised as you would expect, but code written to use Applicative operations benefits from the automatic concurrency. This optimisation is further exploited by using a transformation on the monadic do-notation to automatically use Applicative operators where possible [Marlow et al. 2016]. 
One of the key tools found to be useful in the kind of code written using HaxL at Facebook is the "lazy" conditional operators:

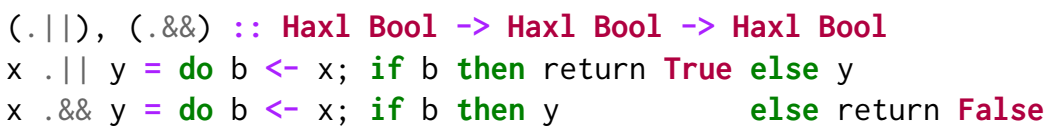

These are typically used to improve performance by guarding slow checks with faster checks. For example, we might write:

if simpleCondition .\&\& complexCondition then ... else ...

The idea is that simpleCondition is quick to evaluate and returns False in a large proportion of cases, so that we can often avoid needing to evaluate complexCondition.

This does not require any additional extensions or special support in HAxL. But we also noticed that sometimes there is a pair of conditions where neither is obviously faster than the other, yet we would still like to benefit from bailing out early when the answer is known. Therefore, HAxL contains two more conditional operators pOr and pAnd for "parallel OR" and "parallel AND":

pOr, pAnd : : Haxl Bool $\rightarrow$ Haxl Bool $\rightarrow$ Haxl Bool

These have the behaviour that: (i) both arguments are evaluated in parallel; (ii) the computation is aborted as soon as the answer is known, even if the other argument is still being evaluated. Data fetches are not observable effects, so the parallelism is not observable to the programmer (HAXL relies on this property for the soundness of its parallel Applicative instance). However, p0r and pAnd are non-deterministic with respect to exceptions: if an exception is thrown by either side, it will be thrown by the computation as a whole immediately without waiting for the other side to complete. One could imagine an alternative implementation which waits for the completion of the other argument when an exception is raised; this would be deterministic, but would be less efficient in the case of exceptions.

It should come as no surprise that pOr and pAnd can be implemented using select, indeed por $=(\langle||\rangle)$ and pAnd $=(\langle \& \&\rangle)$ from Fig. 3 . The corresponding Selective instance is given in Fig. 6: in the Blocked/Blocked case we speculatively explore both computations, and if we obtain a Done/Right result, the second computation is safely abandoned and subsequently cancelled.

There is one wrinkle with implementing pOr and pAnd in terms of select. Ideally, pOr and pAnd would be symmetric: just as we can cancel the second computation if the first one determines the answer, we should be able to cancel the first computation in the same way. Yet select is inherently left-biased: it requires that all the effects of the first argument are performed. In $\$ 6.2$ we consider an alternative combinator related to select that allows this kind of symmetry to be expressed.

We have prototyped an implementation of HAXL with the Selective Haxl instance, which allowed us to reuse generic selective combinators $\langle||\rangle,\langle \& \&\rangle$, anyS and allS instead of providing custom implementations for conditional operators por and pAnd and their generalisations on lists. This case study highlights the fact that selective functors are useful not only in the static context, but in the dynamic context too, by allowing us to benefit from speculative execution.

\subsection{Results}

We mentioned above that pOr and pAnd are effective when the relative size of the conditional computations is unknown, so evaluating them in parallel with early exit is an effective alternative to either sequencing them manually (with Monad) or evaluating them in parallel to completion (with Applicative). This argument becomes even more compelling as the set of conditions to evaluate grows: imagine trying to efficiently sequence a set of ten or more conditions, and then repeating the exercise every time the set changes. 
For this reason, in HAXL we found that list operations built on top of pOr and pAnd, which in this paper we call anyS and alls (see Fig. 3), offer an important balance between performance and maintainability that is not provided by the Applicative or Monad-based combinators.

One could construct examples to demonstrate arbitrarily large performance gains from using por and pAnd, however that would not be particularly useful. Perhaps more useful would be a real-world measurement showing how much performance was improved in an actual application but again, the value of that would depend to a large extent on how the application uses por and pAnd, and unfortunately the application code in our case is proprietary. Therefore instead we offer this anecdote: we first introduced a use of p0r to solve some performance issues in a complex production workload where we had long chains of conditionals that were difficult to optimise by hand, and por resulted in significant performance improvements.

\section{FREE SELECTIVE FUNCTORS}

The idea of describing effectful computations using free constructions, such as free [Swierstra 2008] and freer monads [Kiselyov and Ishii 2015] and free applicative functors [Capriotti and Kaposi 2014] is well-studied in the functional programming community. Free constructions allow us to focus on the internal aspects of the effect under consideration and receive the desired applicative or monadic computation structure for free, i.e. without the need to define custom instances or prove laws.

In this section we apply this idea to selective functors. We present a free construction for rigid selective functors (§5.1), and demonstrate it on two examples in $\S 5.2$ and $§ 5.3$.

\subsection{Free Construction}

In the free structures methodology, the essence of an effect is captured by a data type that encodes the "commands" which the effect provides, acting as a deep embedding of the effect's interface. This data type needs only have enough structure to be a Functor. The purpose of a free construction is then to build a richer structure on top of this base functor, which would have the desired instances, in our case Applicative and Selective. In this section we will denote the base functor by $f$.

As we remarked in $\S 2.3$, rigid selective functors have a particularly simple normal form thanks to the additional law $(\langle *\rangle)=\mathrm{apS}$, which tells us that the apply operator $\langle *\rangle$ is redundant and can be implemented via the selective interface. This normal form has the following linear structure:

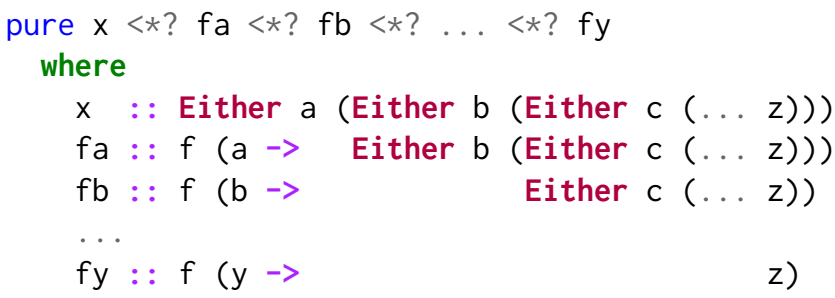

In words, any rigid selective computation can be rewritten as a left-associated sequence of select operators, where the initial pure value $x$ belongs to a large sum type (comprising alternatives a to $z$ in the above snippet), and each of the subsequent effects eliminates one of the alternatives, in order, until only one remains (namely, z).

Interestingly, there is no right-associated version of the normal form because the associativity law (§2.3) can only be used to re-associate an expression to the left, which is a consequence of the asymmetry of the select operator. It is worth noting that this is different from applicative functors that have two normal forms corresponding to left and right re-association of the apply operator [Capriotti and Kaposi 2014]. A symmetric version of the select operator, which can be re-associated in either direction, is discussed in $\S 6.2$. 


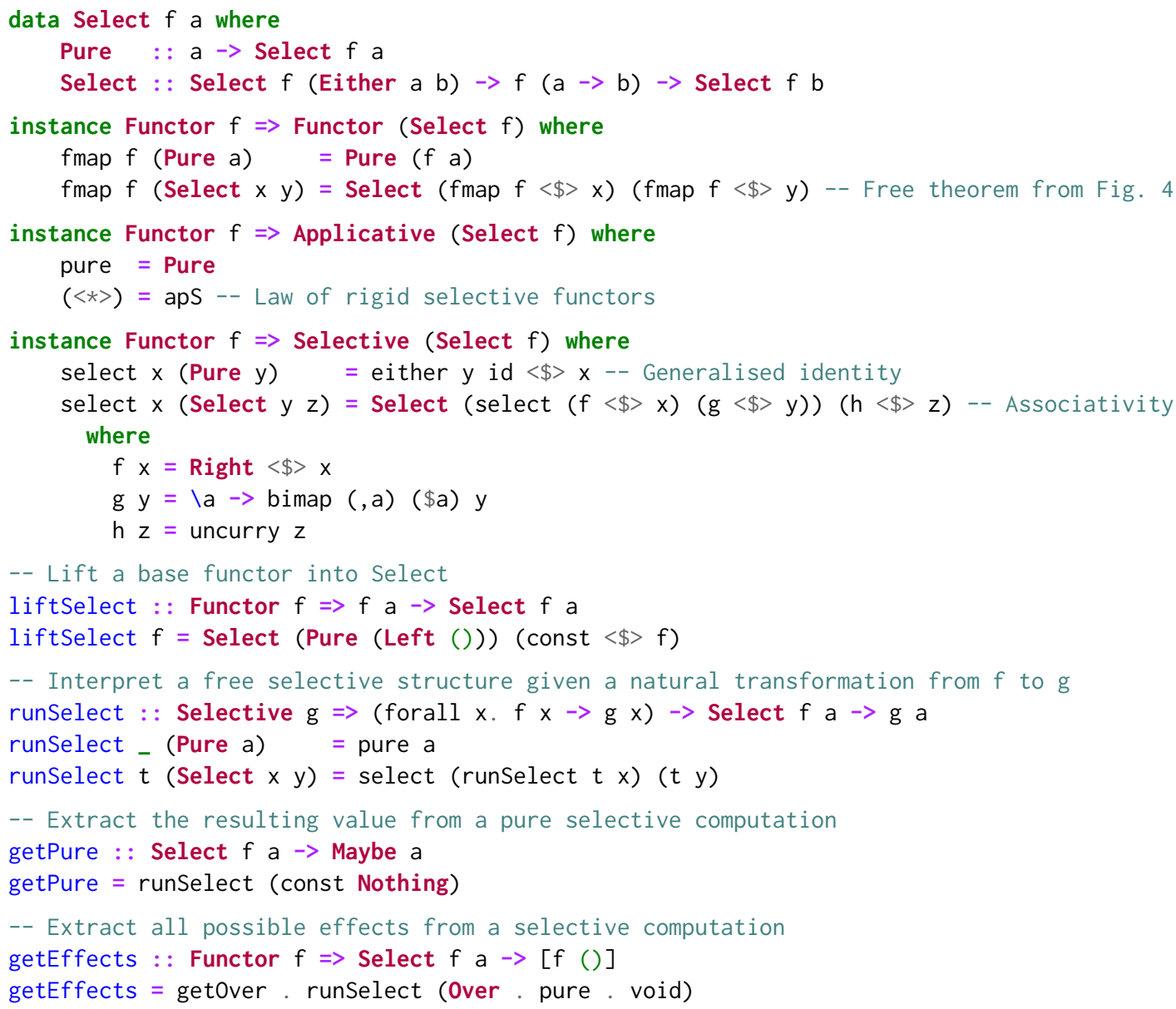

Fig. 7. A basic implementation of free rigid selective functors; various improvements are omitted for clarity.

Fig. 7 gives an encoding of this normal form in Haskell. The free data type Select represents a selective computation as a type-aligned sequence of base functor effects, with the Pure constructor at the head. Instance definitions rely on the selective laws from $§ 2.3$, specifically: generalised identity, associativity, and one of the free theorems. We do not use distributivity as it is subsumed by the law of rigid selective functors $(\langle *\rangle)=$ apS, used in the Applicative instance.

Effects of the base functor can be embedded in the free construction using the helper function liftSelect. To interpret a free selective computation Select $f$ a in a selective functor $g$, one needs to provide a natural transformation from $f$ to $g$ to the function runSelect, which traverses the sequence of effects, converts them to g, and composes the results using g's select operator.

For example, getPure reinterprets a given free computation in the selective functor $g=$ Maybe using the natural transformation const Nothing, which leaves the Pure head of the sequence as is, but turns any subsequent effect into Nothing. Similarly, getEffects records all effects by stashing them in the selective functor Over, which are subsequently extracted from it by getOver.

We can improve the encoding in Fig. 7 in several ways: (i) make it "freer" by not requiring $f$ to be a Functor; (ii) make fmap and select asymptotically faster using the ideas by Menendez [2013]; and (iii) drop the rigidity requirement, obtaining a general free construction for selective functors - see an implementation in the library [Mokhov 2019] and the supplementary material. 


\subsection{Ping-pong, Freely}

To illustrate the usage of free selective functors on a simple example, we implement the classic Teletype DSL [Swierstra 2008] comprising two commands: reading a string form the input stream and writing a string to the output stream. The base functor has two corresponding constructors:

\section{data Teletype $a=$ Read (String $\rightarrow$ a) $\mid$ Write String a deriving Functor}

For convenience, we can provide the following functions that embed the commands into the free selective construction, mimicking Haskell's IO API:

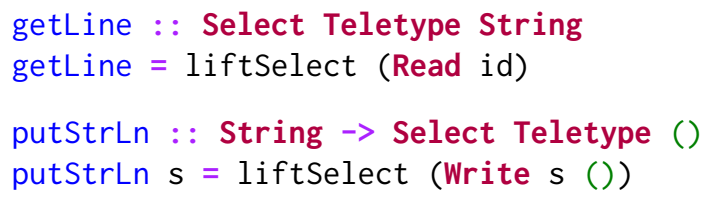

We can now reimplement the pingPongS example from $\S 1$ in terms of the free selective construction simply by adjusting the type signature. Note that the whenS combinator comes for free.

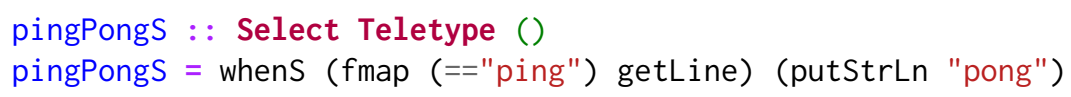

By embedding pingPongS into the free construction, we gain access to the static analysis machinery:

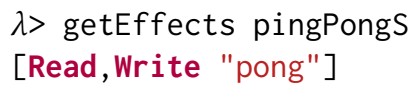

The function getEffects (Fig. 7) returns the list of all effects of a free selective computation. In the case of Teletype, we get a list of all Read/Write commands that a computation might execute.

We can interpret Teletype programs in any other selective functor using the runSelect function by providing a natural transformation forall $\mathrm{x}$. Teletype $\mathrm{x} \rightarrow \mathrm{g} \mathrm{x}$, which assigns an interpretation to Teletype commands in terms of $g$. A good example of such transformation would be an interpretation in the IO monad, which allows us to execute our pingPongS program:

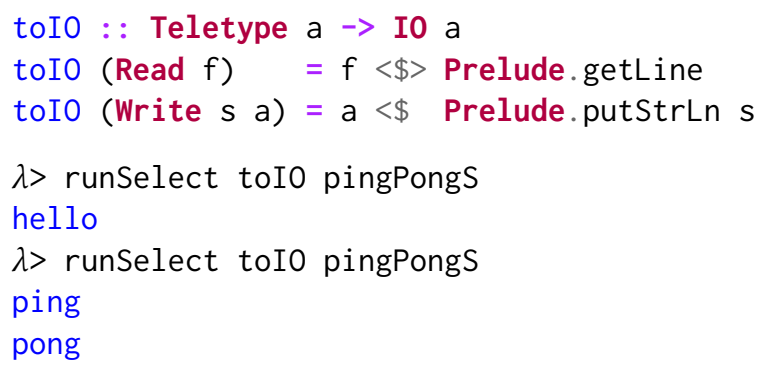

Note that while we can write simple programs like pingPongS using the selective interface, we are fundamentally limited in what we can express compared to the much more powerful monadic interface. As an example, consider this simple greeting program:

$$
\text { greeting = getLine }>>=\text { name }->\text { putStrLn ("Hello " ++ name) }
$$

Programs like this cannot be expressed in our simple Teletype DSL. Even if we had bindS for strings (§6.1), it would be useless for static analysis because it would have to report effects Write s for all possible strings $s$ ! Nevertheless, limitations of the selective interface can sometimes be worked around by using more sophisticated base functors, as we show in $§ 5.3$. 


\subsection{Analysis and Simulation of Processor Instructions}

To demonstrate the free construction on a more interesting example, we apply it to analysis and simulation of a hypothetical instruction set architecture (ISA) ${ }^{6}$. By expressing the ISA semantics in our free construction with an unusual base functor, we will be able to build tools both for static data flow analysis and simulation of programs with branching.

5.3.1 ISA Semantics. To work around some of the aforementioned limitations of the selective interface (namely, the lack of the bind operator), we represent the semantics of instructions thus:

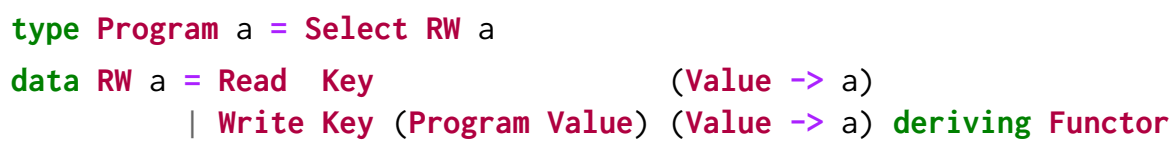

The RW (pronounced "read-write") base functor encodes the effect of a mutable key-value store comprising two commands: (i) we need the ability to read a value associated with a key from the store, and (ii) given a computation which produces a value, write its result into the store. Think of Value as a machine word, and Key as an ISA memory location (a register, a memory cell, or a processor flag). The base commands are similar to Teletype, with one key difference: the Write constructor takes Program Value, i.e. a computation producing a value instead of just plain Value.

This exact structure of the definition is required for accommodating a pattern that occurs frequently in instruction semantics: often we read a value from a register or a memory cell, do something with it, and then write it somewhere else. If Write required the second argument to be a pure value, as in Teletype, we would not be able to express the desired pattern without resorting to the monadic interface. Additionally, we want the Write command to not just write the value and return (), but to give the just written value back, so it can be used in the rest of the computation; such generosity of the Write command will be useful for avoiding duplicate data dependencies.

We introduce two convenience combinators, which lift the data constructors of the RW data type into the free selective, thus making them directly usable in the definitions of instruction semantics:

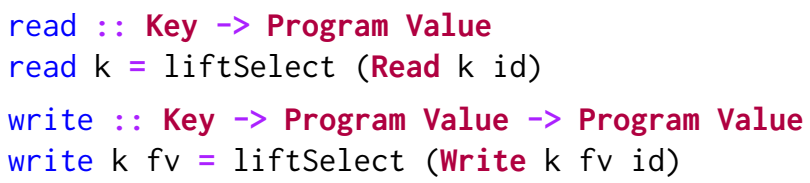

5.3.2 Example 1. Addition. To get acquainted with the introduced vocabulary, we start by describing the semantics for the addition instruction, which reads the summands from a register and a memory cell, adds them, writes the result back into the same register, and also updates the state of the Zero flag to indicate whether the resulting value is zero.

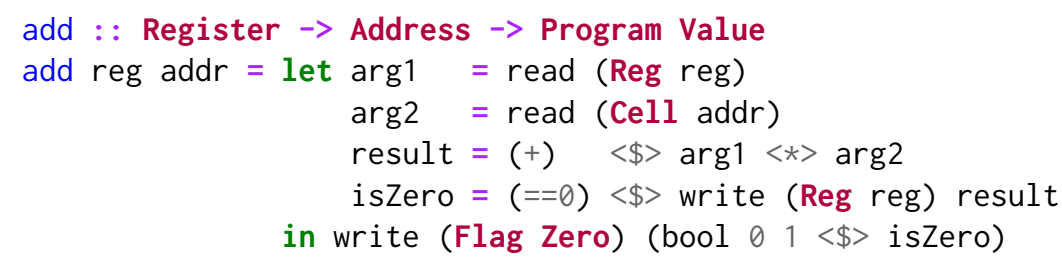

Here, we read the summands arg1 and arg2 from the two specified locations and calculate the result of addition by lifting $(+)$ into the free selective functor using applicative combinators. We

\footnotetext{
${ }^{6}$ Incidentally, this was the original motivation for selective functors. While describing the formal semantics of instructions of a real processor, we needed a statically analysable ifS for the purpose of symbolic program verification, which eventually led us to select. We use a hypothetical ISA in this section instead of the real one, because of the complexity of the latter.
} 
then calculate the value of the Zero flag in a similar way, but here we exploit the fact that the write combinator returns the value it has just written, thus we can reuse the result without recalculating it from scratch (which would duplicate the corresponding read effects).

By analysing the free semantics of the add instruction, we can obtain the list of all its effects, in the order they appear in the computation. We also visualise the effects as a data flow graph, where data locations are shown as rectangles, instructions as rounded rectangles, and reads/writes as arcs.
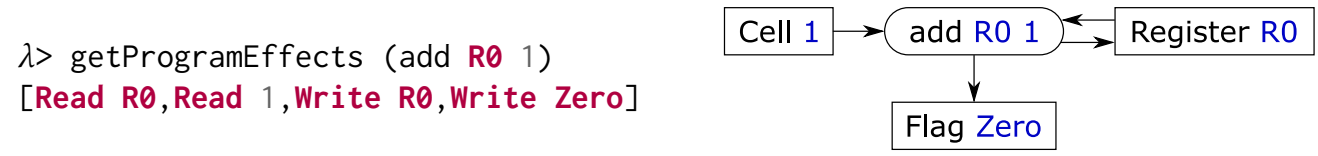

To implement getProgramEffects, we apply the natural transformation toover to the effects of a Program, which recursively collects the effects that occur in the Write's argument fv:

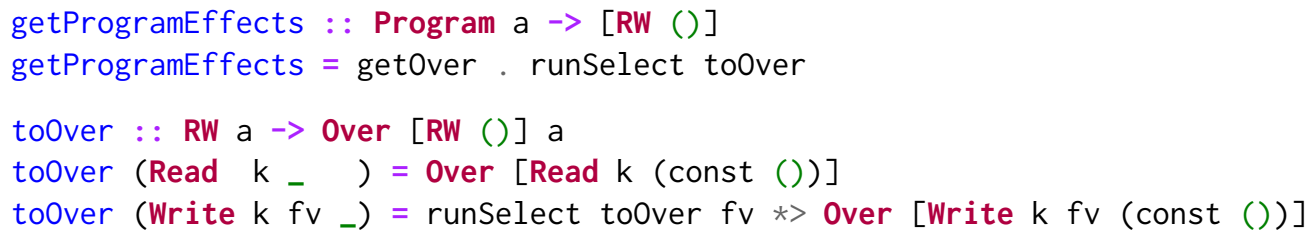

The semantics of the addition instruction has only used applicative combinators and we thus could have analysed it statically using free applicative functors. However, there are important instructions whose semantics cannot be expressed in terms of the Applicative interface, and this is where the presented free selective construction becomes irreplaceable.

5.3.3 Example 2. Conditional Jump. Selective functors introduce limited dependencies between effectful computations, giving us enough power to express the semantics of branching instructions, which modify the program counter by a given offset if a certain condition holds. Consider the following instruction that performs a jump if the result of the previous operation was zero.

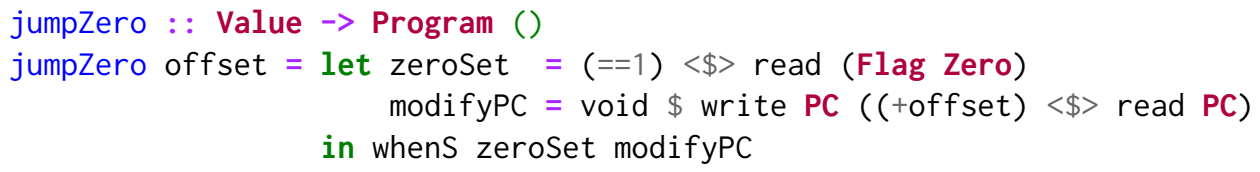

Here we use the whenS combinator to modify the program counter only if the Zero flag is set. By implementing jumpZero in terms of the selective interface, we achieve both the ability to implement an adequate simulator for branching programs and perform their static analysis:

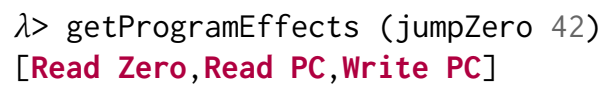

Since the analysis is static, the resulting list of effects and the corresponding data flow graph are over-approximations and show all effects that can possibly happen during the execution.

5.3.4 Example 3. Blocks of Instructions. Once we have implemented the semantics for a desired subset of an ISA, we can describe the semantics of sequences, or blocks, of instructions by simply composing the semantics of individual instructions using the applicative sequencing operator $(*>)$ :

addAndJump : : Program ()

addAndJump $=$ add RO $1 *>$ jumpZero 42 
We can analyse such compound computations in the same way as we analyse individual instructions:

$\lambda>$ getProgramEffects addAndJump
[Read R0, Read 1,Write R0,Write Zero
, Read Zero, Read PC,Write PC]

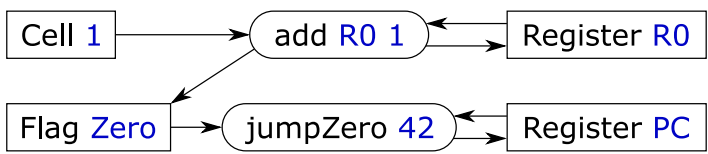

5.3.5 Simulation. To implement an ISA simulator, we follow the same path as in the pingPongS example and the I0 monad earlier in §5.2. We need a natural transformation from the base functor RW to an appropriate target functor, e.g. an instance of MonadState ISAState, where ISAState represents the state of all registers, memory cells and flags. For brevity, we present only one part of such a transformation, which assigns an interpretation to reading and writing of register keys Reg:

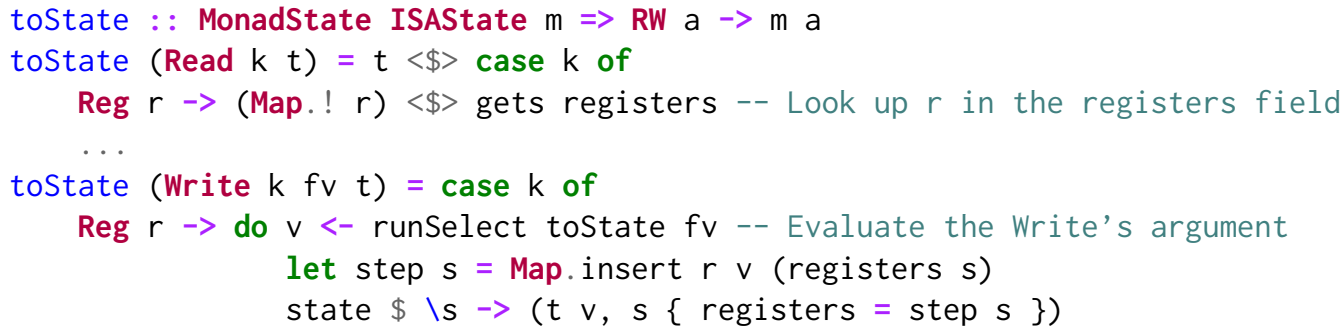

To read a register, we simply lift the lookup function Map.! to the corresponding field of the ISAState. To write an effectful value fv into a register, we need to evaluate it first; hence we call the runSelect function, supplying it the natural transformation toState, recursively, thus performing the effects of $\mathrm{fv}$. We then adjust the register bank with the new value and return it.

The natural transformation toState gives interpretation to individual Read and Write commands, and now this interpretation can be extended to any Program by plugging it into a runSelect call, as has already been done once in the implementation of toState itself:

runProgram :: Program a $\rightarrow$ ISAState $\rightarrow$ (a, ISAState)

runProgram $\mathrm{p}=$ runstate (runselect toState $\mathrm{p}$ )

5.3.6 Limitations. The free selective construction in combination with the base functor RW provides an abstraction capable of expressing the semantics of arithmetic, load/store and branching instructions. However, one should remember that selective functors still lack the full expressive power of the monadic interface and are unable to accommodate an important class of instructions, specifically those that use the memory-indirect addressing mode. If we had a Monad Program instance, we could give the following semantics to the memory-indirect load instruction loadMI:

loadMI : : Register $\rightarrow$ Address $\rightarrow$ Program Value

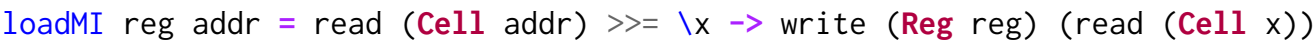

Here, we read from a memory cell addr, then use the monadic bind operator to extract a value $x$ from the result, and use it in a subsequent memory read as address. Although this semantics is, in principle, implementable using the selective bindS combinator (see §6.1), it is not very useful in practice since static analysis would record possible access to all memory cells $\mathrm{x}$, and there are too many of them (typically, a large power of two). Furthermore, the execution of the resulting semantics would be terribly slow, since it would also follow the same linear exploration of the memory address space (although see $§ 6.1$ for a possible solution of the performance issue). 


\section{ALTERNATIVE FORMULATIONS FOR SELECTIVE FUNCTORS}

This section discusses alternative versions of the Selective type class that are based on different select operators: specifically, the multi-way (§6.1) and symmetric (§6.2) generalisations of select, as well as operators that are equivalent to select but may be more convenient to use (§6.3). All of these ideas can be readily integrated into the presented definition of the Selective type class by extending it with new methods and adding new laws that ensure that the new methods interact with select in an appropriate manner. This is common in standard Haskell libraries, where type classes Applicative and Monad include methods like $*>$ and $>>$ for performance reasons.

Another alternative, which is worth a remark, is to simply add select to the Applicative type class, with the default implementation select = selectA. While this works for the purposes discussed in this paper, it would make it harder to reason about code with the Applicative $f$ constraint, since the select method makes it possible for effects to depend on values; declaring such a significant ability by the Selective $f$ constraint is arguably a more prudent approach.

\subsection{Multiway Selective Functors}

As mentioned in $\S 2$, branch is a strong contender to be the main method of the Selective type class; it is parametric and all selective combinators, including select itself, can be derived from it:

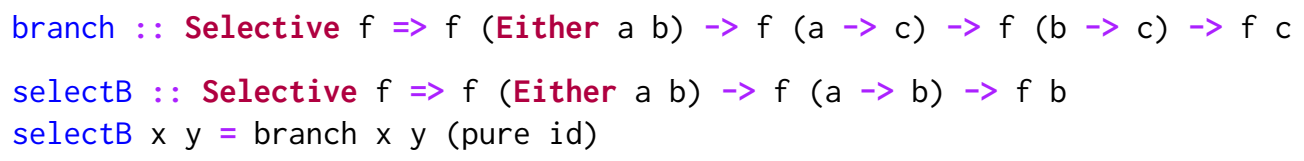

While we prefer select for its simplicity, branch does provide an interesting advantage in the context of static analysis. Specifically, it makes it statically apparent that the two branches are mutually exclusive. When branch is "desugared" into a sequence of two select operations, the information about the mutual exclusion between the two branches is lost, which rules out some static analysis scenarios. For example, it may be useful to know that in our build systems example in $\S 3.2$ we never depend on both lib.c and lib.ml.

Another point in favour of branch is performance: the select-based implementation of the ifS combinator checks for the Left and Right cases in sequence, instead of directly jumping to the correct case, so a branch-based implementation would be more efficient. Furthermore, $N$-way generalisations of select are possible, although the design space here is quite large. As an example, one might consider adding bindS to the Selective type class, i.e. a special case of the monadic bind operator that is applicable only to enumerable types:

bindS : : Selective $f \Rightarrow$ (Bounded $a$, Enum $a$, Eq $a) \Rightarrow f a \rightarrow(a \rightarrow f b) \rightarrow f b$

The default implementation could be based on sequentially checking for every possible value using select, but monadic instances would supply a much faster implementation, namely bindS = (>>=). This would allow static analysis instances to record all possible cases, without incurring the $O(N)$ slowdown during the execution of an $N$-way branch.

Interestingly, adding the ability to branch on infinite number of cases makes selective functors equivalent to monads, e.g. see Peebles [2019]. However, it is worth pointing out that static analysis of such infinitely-branching selective functors might take infinite time too.

Exploring the design space for "multiway selective functors", and using them for efficient translation of Haskell's do-notation into selective combinators in the spirit of the ApplicativeDo extension [Marlow et al. 2016] is left for future research. For now, we believe that adding branch and/or an equivalent of bindS to the Selective type class would be beneficial for performancesensitive applications. 


\subsection{Symmetric Selective Functors}

In this section we address the asymmetry of select, which we remarked on in $\S 4$ and $\S 5$. The asymmetry can be seen in the fact that the first argument of select must always be executed, while the second argument may sometimes be skipped. Consider a more symmetric alternative:

$$
\text { biselect : Selective } f \Rightarrow f(\text { Either } a b) \rightarrow f(\text { Either } a c) \rightarrow f(\text { Either } a(b, c) \text { ) }
$$

This definition is pleasantly symmetric: if either of the arguments yields a Left a value, the other argument may be skipped since the result must be a Left a too, by parametricity. On the other hand, if one of the arguments yields a Right value, then the other argument must be executed in order to either get an a or the other half of the resulting pair. As an added bonus, the rather obscure associativity law from $§ 2.3$ looks much more natural for $($ ?*?) = biselect:

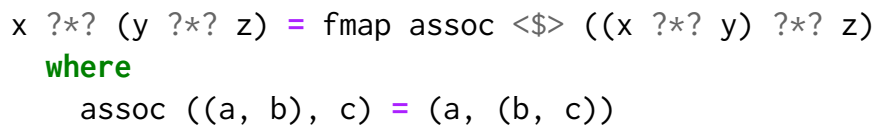

While beautiful, we found biselect to be a bit more awkward to work with than select, and also more subtle when the order in which the arguments are executed is not fixed. So far we have identified only one example where the symmetry of biselect is beneficial: speculative execution of parallel OR and AND combinators - see the HAXL case study $\$ 4$. To support such use-cases it is possible to add biselect to the Selective type class with the following default implementation:

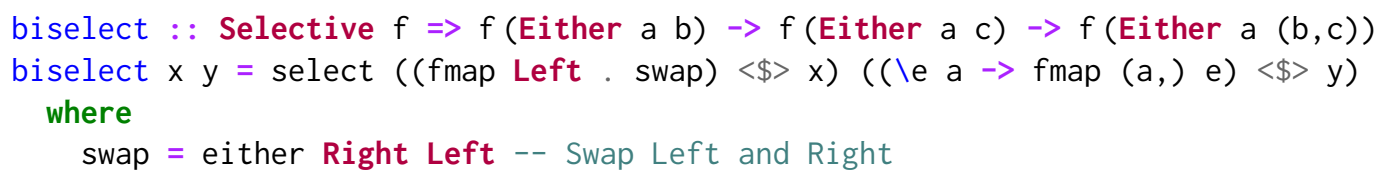

This implementation breaks the symmetry, which may be acceptable for most instances of selective functors, but instances like Haxl would override it in order to gain additional performance benefits. Note that the selective combinators like $\langle||\rangle$ would need to be redefined via biselect in order to take advantage of the symmetry.

From the theoretical viewpoint, the type signature of biselect makes it more apparent that a selective functor $f$ is a composition of an applicative functor $f$ and the Either monad.

\subsection{Equivalent Formulations}

In this section we briefly mention three equivalent operators that can be used instead of select.

- Lennox S. Leary and Edward Kmett suggested to move the function to the first argument: select : : Selective $f \Rightarrow f$ (Either $(a-b) b) \rightarrow f a \rightarrow f b$

This operator is similar to Applicative's $\langle *\rangle$ but with a twist: the first argument might turn out to be a constant function const $b$, in which case the effect $f$ a may be skipped.

- One can take one step further and extract the selection logic into a separate function: selectBy : : Selective $f \Rightarrow(a \rightarrow$ Either $(b \rightarrow c) c) \rightarrow f a \rightarrow f b \rightarrow f c$ This operator is very convenient for implementing selective combinators, and also provides more opportunities for optimisation by fusing construction and deconstruction of Either's.

- Finally, it is possible to get rid of functions altogether: select : : Selective $f \Rightarrow f$ (Either $a b) \rightarrow f c \rightarrow f$ (Either $a(b, c)$ )

While this formulation requires an extra tuple allocation, it uses only sum and product types, and may therefore be useful in contexts where functions are unavailable. 


\section{RELATED WORK}

Composing effectful computations is a rich research area and there is a vast body of related work. We build on the fundamental notions of applicative functors [McBride and Paterson 2008] and monads [Moggi 1991; Wadler 1995], but these notions are not isolated: the space between them is inhabited by arrows [Hughes 2000] and generalised arrows [Megacz 2011], which we discuss in §7.1.

The idea of extending the Applicative interface to gain more expressive power is not new. Parser combinators by Swierstra and Duponcheel [1996] paved the way to the Alternative type class ( $\$ 7.2)$. Yallop [2010] proposed to extend Applicative with a method of type $f$ Bool $\rightarrow f$ a $\rightarrow f$ a $\rightarrow f$ a "for capturing computations where control flow is dynamic, but dataflow is static"; similar ideas were studied by hardware designers in the context of synchronous [Dennis and Misunas 1975] and asynchronous [Mokhov 2009; Sokolov et al. 2018] control circuits. We have also found early online discussions [Permyakov et al. 2012; Yorgey et al. 2009] that searched for type classes like Selective but did not progress further. ApplicativeFix proposed by Devriese et al. [2013] can be combined with selective functors to allow for static analysis of effectful computations with cycles.

Many selective combinators appeared earlier on an ad hoc basis, including HaxL's speculative execution functions p0r and pAnd $(\S 4)$, the "parallel conjunction" operator $(* \& *)$ in Lazy SmallCheck [Runciman et al. 2008], and various examples of the if statement using special optimisations instead of relying on the monadic interface [Jane Street 2015]. Finally, the type signature of select resembles an exception handler [Benton and Kennedy 2001] where the first argument may raise an exception to be handled by the second argument - this explains why the initial blog post exploring selective functors used handle as the operator name instead of select [Mokhov 2018].

Our free construction for rigid selective functors $(\S 5)$ is inspired by the works on free applicative functors [Capriotti and Kaposi 2014], free monads [Swierstra 2008], and insightful blog posts by Fancher [2016, 2017]. Batching and remote execution of effectful computations [Gill et al. 2015] can be greatly simplified by using free applicative functors, as demonstrated by Gibbons [2016], and we believe that free selective functors uncover new opportunities in this area.

\subsection{Arrows and Profunctors}

Arrows, introduced by Hughes [2000], generalise functors by making the input of a computation explicit. Rather than giving the type $f$ a to an effectful computation that yields a value of type a, as we have done in this paper so far, arrows give the type a i o to an effectful computation that takes values of type $i$ as input and yields values of type $o$ as output. There is a rich arrow hierarchy of type classes, each providing a new ability, where ArrowChoice is particularly relevant for us:

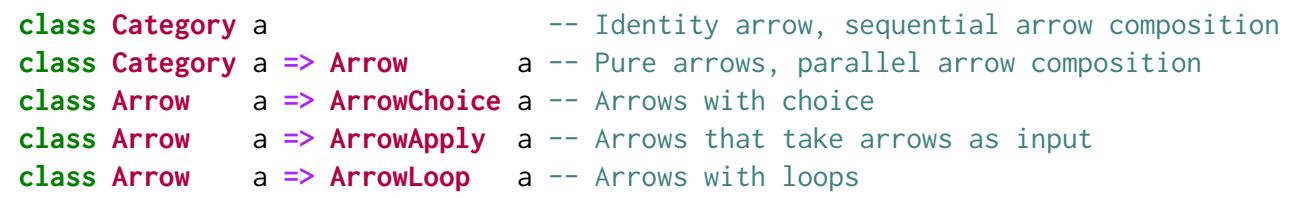

The relationships between applicative functors, monads and arrows have been studied in depth. It is known, e.g. see Lindley et al. [2011] and Rivas and Jaskelioff [2017], that applicative functors correspond to so called static arrows, for which there is an isomorphism between a () ( $->0)$ and $\mathrm{a} i \mathrm{o}$. The standard module Control.Arrow therefore provides the following definitions:

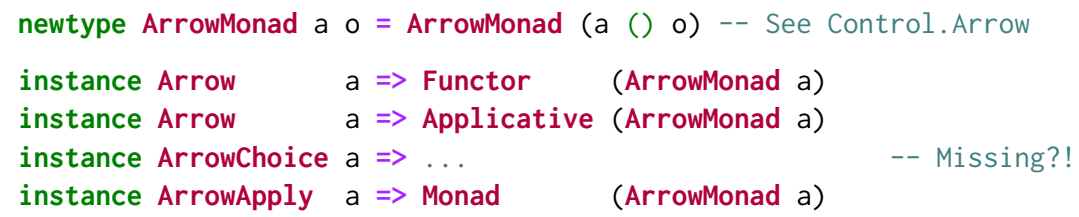


Selective functors provide the missing counterpart for ArrowChoice in the functor hierarchy, as demonstrated by the following instance:

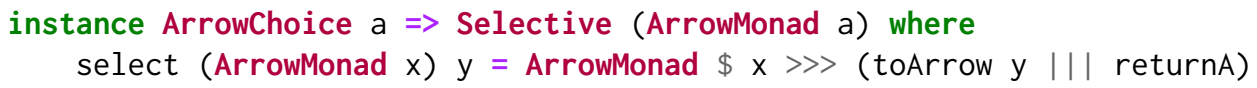

Here toArrow witnesses one half of the aforementioned isomorphism between a () ( $i->0)$ and a $\mathrm{i}$ o. The obtained Selective instance is lawful thanks to the ArrowChoice laws.

Arrows are more general and powerful than selective functors. We could have used arrows to solve our static analysis and speculative execution examples, and not just in theory - DuNE is a great example of successful application of arrows in practice. However, introducing arrows to an existing codebase built around applicative functors and monads, such as HAXL, would require pervasive changes to the whole abstraction stack, as well as rewriting all existing HAxL user code in the arrow notation [Paterson 2001]. Needless to say, introduction of selective functors to HAXL is a much easier task, which we have accomplished by adding 13 lines of new code for the definition of the Selective Haxl instance, and removing 26 lines of code corresponding to similarly-sized definitions of pOr and pAnd, reusing the selective combinators $\langle||\rangle$ and $\langle \& \&\rangle$ instead.

Profunctors is an abstraction closely related to arrows; see [Pickering et al. 2017] for a good overview of profunctors in the context of modular data accessors, or lenses. Similarly to ArrowChoice, so-called Cocartesian profunctors are counterparts of selective functors in the profunctor hierarchy.

Establishing a formal correspondence between ArrowChoice, Cocartesian profunctors, and selective functors is beyond the scope of this paper and is left for future research.

\subsection{Parser Combinators}

Alternative is a type class originally motivated by non-monadic parsers; see, for example, Swierstra and Duponcheel [1996], where the methods of the Alternative type class appear as part a bigger Parsing type class. In modern Haskell, Alternative is a subclass of Applicative:

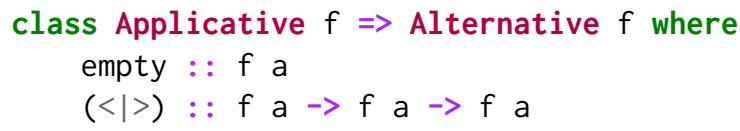

The operator $<\mid>$ allows us to naturally express choice in parsers. As an example, consider the task of parsing binary and hexadecimal numbers, which are prefixed with " $0 b "$ and " $0 x "$, respectively. Following the classic parser combinator approach [Hutton and Meijer 1998], let us assume the existence of the following parsers:

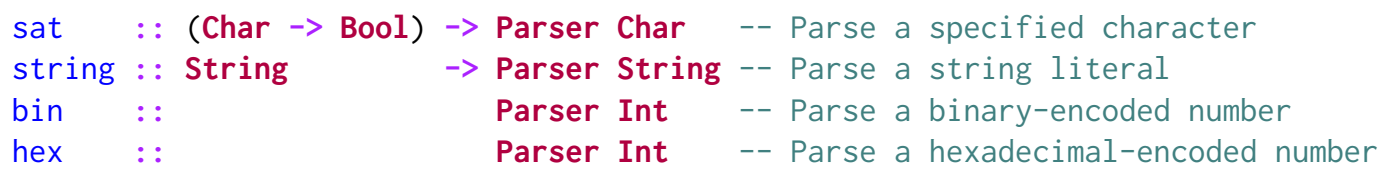

Now the desired parser can be obtained as a choice between parsers for binary and hexadecimal numbers, each augmented with the prefix-parsing part:

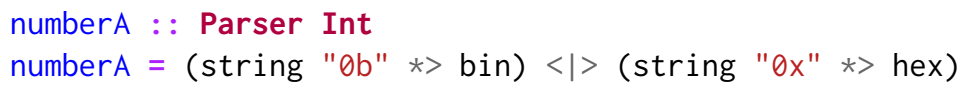


When parsing "Ox7E3", the first parser fails (due to the prefix mismatch), but the second one succeeds. Note that parsing of the leading " 0 " can be factored out into a separate parser string " $\theta$ " to avoid backtracking.

Selective functors also allow us to implement the desired parser, and arguably in a more direct style that does not involve trying one parser after another:

numberS : : Parser Int

numberS = string " $0 " *\rangle$ ifS $\left(\left({ }^{\prime} b^{\prime}==\right)\langle \$\rangle\right.$ sat (`elem` "bx")) bin hex

Here we first parse the leading " $\theta$ ", then the second character of the prefix, failing if it is neither "b" nor " $x$ ", and finally select an appropriate subsequent parser using ifS. Note that we can move the parser string " 0 " in and out of the condition ifS thanks to the interchange law (§2.3).

Investigation of the relationship between Alternative and Selective type classes, as well as application of selective functors to parsers is an interesting research opportunity.

\section{CONCLUSIONS}

We have introduced selective functors, an abstraction between applicative functors and monads. Like applicative functors, selective functors require all effects to be known statically, before the execution starts. Like monads, selective functors allow for effects to depend on values of earlier effects but in a limited way: it is possible to skip some of the effects, but not create new ones. In this sense selective functors allow you to describe computations that are very much like hardware circuits: statically fixed, yet dynamically reconfigurable.

We have demonstrated usefulness of the new abstraction on several examples, and hope that the reader will find it useful in their next project too.

\section{ACKNOWLEDGMENTS}

We are very grateful to everyone who contributed by participating in numerous discussions and providing feedback on earlier drafts of this paper.

Arseniy Alekseyev, Ulan Degenbaev and Neil Mitchell have been closely following the work on selective functors from the very first blog post; their early and constructive feedback encouraged and guided our research.

Many others have joined and helped as the work progressed, including: Thorsten Altenkirch, Baldur Blöndal, Dominique Devriese, Ivan Gotovchits, Oleg Grenrus, Jennifer Hackett, Graham Hutton, Luka Jacobowitz, Edward Kmett, Lennox S. Leary, Gábor Lehel, Sam Lindley, Tim McGilchrist, James McKinna, Yaron Minsky, Alexandre Moine, Matthew Naylor, Daniel Peebles, Artem Pelenitsyn, Simon Peyton Jones, Ivan Polyakov, Gabriel Radanne, Asad Saeeduddin, Irakli Safareli, Carter Schonwald, Danil Sokolov, Ian Treyball, Anton Trunov, Cristian Urlea, Sjoerd Visscher, Alexa de Wit, Brent Yorgey, Vladislav Zavialov, and reddit users Darwin226, dmwit, sclv, viercc and yakrar. With such an active and helpful community, we are certain that the above list is just an under-approximation of all our interactions, and we apologise for any omissions.

Last but not least, we would like to thank the four ICFP reviewers who discovered and helped to fix a few important issues in the submitted version of the paper.

Andrey Mokhov's research is supported by a Royal Society Industry Fellowship IF160117 on the topic "Towards Cloud Build Systems with Dynamic Dependency Graphs". 


\section{REFERENCES}

Nick Benton and Andrew Kennedy. 2001. Exceptional syntax. fournal of Functional Programming 11, 4 (2001), 395-410.

Yves Bertot and Pierre Castéran. 2013. Interactive theorem proving and program development: Coq'Art: the calculus of inductive constructions. Springer Science \& Business Media.

Chris Birchall and Hamish Dickson. 2019. Implementation of selective applicative functors in Scala. (2019). https://web.archive.org/web/20190623214126/https://github.com/cb372/cats-selective/blob/master/core/src/main/ scala/cats/Selective.scala.

Baldur Blöndal, Andres Löh, and Ryan Scott. 2018. Deriving Via. In Proceedings of the 11th ACM Haskell Symposium (Haskell'18).

Paolo Capriotti and Ambrus Kaposi. 2014. Free applicative functors. Proceedings 5th Workshop on Mathematically Structured Functional Programming 153, 2-30.

Jack B Dennis and David P Misunas. 1975. A preliminary architecture for a basic data-flow processor. In ACM SIGARCH Computer Architecture News, Vol. 3. ACM, 126-132.

Dominique Devriese, Ilya Sergey, Dave Clarke, and Frank Piessens. 2013. Fixing idioms: a recursion primitive for applicative DSLs. In Proceedings of the ACM SIGPLAN 2013 workshop on Partial evaluation and program manipulation. ACM, 97-106.

Will Fancher. 2016. More on Applicative Effects in Free Monads. (2016). https://web.archive.org/web/20190307232337/https: //elvishjerricco.github.io/2016/04/13/more-on-applicative-effects-in-free-monads.html.

Will Fancher. 2017. Profunctors, Arrows, \& Static Analysis. (2017). https://web.archive.org/web/20190307232429/https: //elvishjerricco.github.io/2017/03/10/profunctors-arrows-and-static-analysis.html.

Jeremy Gibbons. 2016. Free delivery (functional pearl). In ACM SIGPLAN Notices, Vol. 51. ACM, 45-50.

Richard Gibson. 2019. Implementation of selective applicative functors in Kotlin. (2019). https://web. archive.org/web/20190623213927/https://github.com/arrow-kt/arrow/blob/master/modules/core/arrow-coredata/src/main/kotlin/arrow/typeclasses/Selective.kt.

Andy Gill, Neil Sculthorpe, Justin Dawson, Aleksander Eskilson, Andrew Farmer, Mark Grebe, Jeffrey Rosenbluth, Ryan Scott, and James Stanton. 2015. The remote monad design pattern. In ACM SIGPLAN Notices, Vol. 50. ACM, 59-70.

Robert Harper. 2011. Boolean Blindness. (2011). https://web.archive.org/web/20110321191234/http://existentialtype. wordpress.com/2011/03/15/boolean-blindness/.

Antti Holvikari. 2018. Implementation of selective applicative functors in PureScript. (2018). https://web.archive.org/web/ 20190623214040/https://github.com/anttih/purescript-selective/blob/master/src/Control/Selective.purs.

John Hughes. 2000. Generalising monads to arrows. Science of computer programming 37, 1-3 (2000), 67-111.

Graham Hutton and Erik Meijer. 1998. Monadic parsing in Haskell. fournal of functional programming 8, 4 (1998), $437-444$.

Jane Street. 2015. Incremental: A library for incremental computations. Bind, scopes, and invalidation (2015). https://web.archive.org/web/20190709231158/https://ocaml.janestreet.com/ocaml-core/latest/doc/incremental/ Incremental_/Incremental_intf/\#bind,-scopes,-and-invalidation.

Jane Street. 2018. Dune: A composable build system. (2018). https://dune.build/.

Oleg Kiselyov and Hiromi Ishii. 2015. Freer Monads, More Extensible Effects. SIGPLAN Not. 50, 12 (Aug. 2015), 94-105.

Daan Leijen and Erik Meijer. 2000. Domain specific embedded compilers. ACM Sigplan Notices 35, 1 (2000), 109-122.

Sam Lindley, Philip Wadler, and Jeremy Yallop. 2011. Idioms are oblivious, arrows are meticulous, monads are promiscuous. Electronic notes in theoretical computer science 229, 5 (2011), 97-117.

Georgy Lukyanov. 2019. Implementation of selective applicative functors in Coq. (2019). https://web.archive.org/web/ 20190623213821/https://github.com/tuura/selective-theory-coq/blob/master/src/Control/Selective.v.

Simon Marlow, Louis Brandy, Jonathan Coens, and Jon Purdy. 2014. There is no fork: An abstraction for efficient, concurrent, and concise data access. In ACM SIGPLAN Notices, Vol. 49. ACM, 325-337.

Simon Marlow, Simon Peyton Jones, Edward Kmett, and Andrey Mokhov. 2016. Desugaring Haskell's do-notation into applicative operations. In ACM SIGPLAN Notices, Vol. 51. ACM, 92-104.

Conor McBride and Ross Paterson. 2008. Applicative programming with effects. Fournal of Functional Programming 18, 1 (2008), 1-13.

Adam Megacz. 2011. Hardware design with generalized arrows. In International Symposium on Implementation and Application of Functional Languages. Springer, 164-180.

Dave Menendez. 2013. Free Applicative Functors in Haskell. (2013). https://web.archive.org/web/20190625202450/https: //www.eyrie.org/ zednenem/2013/05/27/freeapp.

Eugenio Moggi. 1991. Notions of computation and monads. Information and computation 93, 1 (1991), 55-92.

Andrey Mokhov. 2009. Conditional partial order graphs. Ph.D. Dissertation. Newcastle University.

Andrey Mokhov. 2018. Selective applicative functors (blog post). (2018). https://web.archive.org/web/20190501160729/https: //blogs.ncl.ac.uk/andreymokhov/selective/.

Andrey Mokhov. 2019. Implementation of selective applicative functors in Haskell. (2019). https://hackage.haskell.org/ package/selective. 
Andrey Mokhov, Neil Mitchell, and Simon Peyton Jones. 2018. Build Systems à la Carte. Proceedings of the ACM on Programming Languages 2, ICFP (2018), 79.

Ross Paterson. 2001. A new notation for arrows. In ACM SIGPLAN Notices, Vol. 36. ACM, 229-240.

Daniel Peebles. 2019. Sigma Selective. (2019). http://web.archive.org/web/20190625225137/https://gist.github.com/ copumpkin/d5bdbc7afda54ff04049b6bdbcffb67e.

Evgeny Permyakov et al. 2012. Applicative functors with branch/choice. (2012). https://web.archive.org/web/20190626004303/ https://mail.haskell.org/pipermail/haskell-cafe/2012-July/102518.html.

Matthew Pickering, Jeremy Gibbons, and Nicolas Wu. 2017. Profunctor optics: Modular data accessors. Art, Science, and Engineering of Programming 1, 2 (2017).

Exequiel Rivas and Mauro Jaskelioff. 2017. Notions of computation as monoids. Fournal of functional programming 27 (2017).

Tomás Ruiz-López. 2019. Implementation of selective applicative functors in Swift. (2019). https://web.archive.org/save/https: //github.com/bow-swift/bow/blob/master/Sources/Bow/Typeclasses/Selective.swift.

Colin Runciman, Matthew Naylor, and Fredrik Lindblad. 2008. SmallCheck and Lazy SmallCheck: automatic exhaustive testing for small values. In Acm sigplan notices, Vol. 44. ACM, 37-48.

Danil Sokolov, Alessandro de Gennaro, and Andrey Mokhov. 2018. Reconfigurable asynchronous pipelines: From formal models to silicon. In 2018 Design, Automation \& Test in Europe Conference \& Exhibition (DATE). IEEE, 1562-1567.

S Doaitse Swierstra and Luc Duponcheel. 1996. Deterministic, error-correcting combinator parsers. In International School on Advanced Functional Programming. Springer, 184-207.

Wouter Swierstra. 2008. Data types à la carte. Journal of functional programming 18, 4 (2008), 423-436.

The OPAM team. 2018. OCaml Package Manager. (2018). https://opam.ocaml.org/.

Philip Wadler. 1989. Theorems for free!. In FPCA, Vol. 89. 347-359.

P. Wadler. 1995. Monads for functional programming. In Int'l School on Advanced Functional Programming. Springer, 24-52. Jeremy Yallop. 2010. Abstraction for web programming. Ph.D. Dissertation. The University of Edinburgh.

Brent Yorgey et al. 2009. An IRC conversation about Branchy type class. (2009). https://web.archive.org/web/20190626003950/ https://github.com/snowleopard/selective/blob/master/paper/irc-log-branchy.md. 